\title{
FLAT OUTPUTS OF TWO-INPUT DRIFTLESS CONTROL SYSTEMS
}

\author{
SHUN-JiE Li ${ }^{1}$ AND Witold RESPONDEK ${ }^{1}$
}

\begin{abstract}
We study the problem of flatness of two-input driftless control systems. Although a characterization of flat systems of that class is known, the problems of describing all flat outputs and of calculating them is open and we solve it in the paper. We show that all $x$-flat outputs are parameterized by an arbitrary function of three canonically defined variables. We also construct a system of 1st order PDE's whose solutions give all $x$-flat outputs of two-input driftless systems. We illustrate our results by describing all $x$-flat outputs of models of a nonholonomic car and the $n$-trailer system.
\end{abstract}

Mathematics Subject Classification. 93B27, 93C10, 93C95.

Received November 18, 2010.

Published online October 14, 2011.

\section{INTRODUCTION}

The notion of flatness has been introduced by Fliess et al. [5-7] in order to describe the class of control systems whose set of trajectories can be parameterized by a finite number of functions and their time-derivatives. More formally, a system with $m$ controls is flat if we can find $m$ functions (of the state and control variables and their time-derivatives), called flat outputs, such that the evolution in time of the state and control can be expressed in terms of flat outputs and their time derivatives (see Sect. 1 for a precise definition and references).

As an introductory example, consider the nonholonomic car or, equivalently, a unicycle-like robot towing a trailer (see, e.g., [16]), shown in Figure 1. Denote by $(x, y) \in \mathbb{R}^{2}$ the position of the mid-point of the rear wheels, and by $\theta_{0}$ and $\theta_{1}$, respectively, the angles between the front and rear wheels and the $x$-axis. The controls $u_{1}$ and $u_{2}$ allow to move (forward and backward) the car and to turn. The car is subject to two nonholonomic constraints: the wheels are not allowed to slide. This leads to the following model given by a driftless, i.e., control-linear, system on $\mathbb{R}^{2} \times S^{1} \times S^{1}$ :

$$
\Sigma_{\text {car }}:\left(\begin{array}{c}
\dot{x} \\
\dot{y} \\
\dot{\theta}_{0} \\
\dot{\theta}_{1}
\end{array}\right)=\left(\begin{array}{c}
\cos \left(\theta_{1}-\theta_{0}\right) \cos \theta_{0} \\
\cos \left(\theta_{1}-\theta_{0}\right) \sin \theta_{0} \\
\sin \left(\theta_{1}-\theta_{0}\right) \\
0
\end{array}\right) u_{1}+\left(\begin{array}{l}
0 \\
0 \\
0 \\
1
\end{array}\right) u_{2} .
$$

Keywords and phrases. Control system, flatness, flat output, feedback equivalence, characteristic distribution, $n$-trailer system.

${ }^{1}$ INSA-Rouen, Laboratoire de Mathématiques, Avenue de l'Université, 76801 Saint-Etienne-du-Rouvray, France.

shunjie.li@insa-rouen.fr; witold.respondek@insa-rouen.fr 


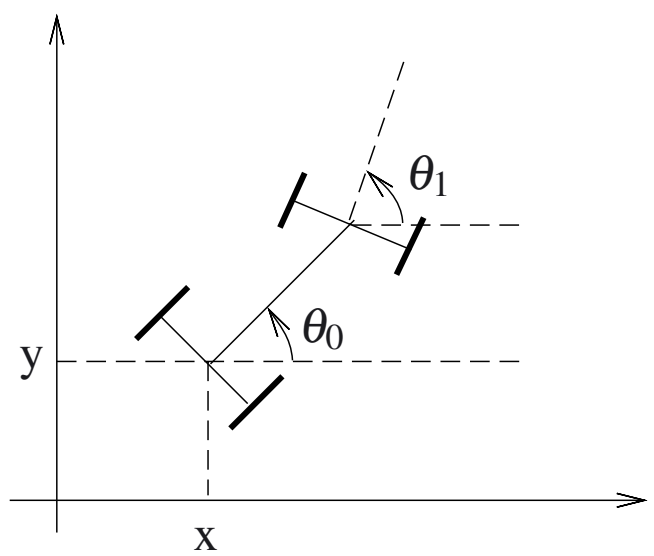

Figure 1. The nonhonomic car.

It is well known, as proved by Fliess et al. [6] (see also [13]), that the nonholonomic car is flat and that the position $(x, y)$ of the mid-point of the rear wheels is a flat output. Indeed, the following coordinates change

$$
\begin{aligned}
& x_{1}=x \\
& x_{2}=y \\
& x_{3}=\tan \theta_{0} \\
& x_{4}=\tan \left(\theta_{1}-\theta_{0}\right) \sec ^{3} \theta_{0}
\end{aligned}
$$

for $\theta_{0} \in\left(-\frac{\pi}{2}, \frac{\pi}{2}\right)$ and $\theta_{1}-\theta_{0} \in\left(-\frac{\pi}{2}, \frac{\pi}{2}\right)$, followed by the invertible feedback

$$
\begin{aligned}
& v_{1}=u_{1} \cos \left(\theta_{1}-\theta_{0}\right) \cos \theta_{0} \\
& v_{2}=u_{1} \sec ^{3} \theta_{0} \sin \left(\theta_{1}-\theta_{0}\right)\left(3 \tan \left(\theta_{1}-\theta_{0}\right) \tan \theta_{0}-\sec ^{2}\left(\theta_{1}-\theta_{0}\right)\right)+u_{2} \sec ^{3} \theta_{0} \sec ^{2}\left(\theta_{1}-\theta_{0}\right),
\end{aligned}
$$

brings the system $\Sigma_{\text {car }}$ into the chained form:

$$
\left\{\begin{array}{l}
\dot{x}_{1}=v_{1} \\
\dot{x}_{2}=x_{3} v_{1} \\
\dot{x}_{3}=x_{4} v_{1} \\
\dot{x}_{4}=v_{2}
\end{array}\right.
$$

It is easy to see that the pair of functions $h=\left(h_{1}, h_{2}\right)=\left(x_{1}, x_{2}\right)=(x, y)$ are flat outputs for the chained form. Indeed, we have $x_{1}=h_{1}, x_{2}=h_{2}, x_{3}=\frac{\dot{h}_{2}}{\dot{h}_{1}}, x_{4}=\frac{1}{\dot{h}_{1}} \frac{\mathrm{d}}{\mathrm{d} t}\left(\frac{\dot{h}_{2}}{\dot{h}_{1}}\right), v_{1}=\dot{h}_{1}$ and $v_{2}=\dot{x}_{4}$. The applied transformation, consisting of a change of coordinates and feedback, is invertible which proves that, indeed, $h=(x, y)$ is a flat output of the nonholonomic car.

The presented procedure, to express the state and control in terms of $h$ and its time-derivatives, exhibits three singularities: two in the state space (at $\theta_{0}= \pm \frac{\pi}{2}$ and $\theta_{1}-\theta_{0}= \pm \frac{\pi}{2}$ ) and one in the control space (when $\left.u_{1}(t)=v_{1}(t)=0\right)$. Let us analyze those singularities.

First, the singularity at $u_{1}(t)=0$ (at least at $u_{1}(t) \equiv 0$ ) seems to be intrinsic: we cannot see how the angles $\theta_{0}(t)$ and $\theta_{1}(t)$ evolve if the observed point $(x(t), y(t))$ does not move. 
Secondly, $\theta_{0}= \pm \frac{\pi}{2}$ is not a singularity of the flat output $h=(x, y)$. Indeed, around a point such that $\theta_{0}= \pm \frac{\pi}{2}$, we can choose the following coordinates change:

$$
\begin{aligned}
& x_{1}=y \\
& x_{2}=x \\
& x_{3}=\cot \theta_{0} \\
& x_{4}=-\tan \left(\theta_{1}-\theta_{0}\right) \csc ^{3} \theta_{0},
\end{aligned}
$$

for $\theta_{0} \in(0, \pi)$, including $\theta_{0}=\frac{\pi}{2}$ (or $\theta_{0} \in(-\pi, 0)$, including $\left.\theta_{0}=-\frac{\pi}{2}\right)$ and $\theta_{1}-\theta_{0} \in\left(-\frac{\pi}{2}, \frac{\pi}{2}\right)$, followed by a suitable feedback transformation, which brings the system $\Sigma_{\text {car }}$ into the chained form around $\theta_{0}= \pm \frac{\pi}{2}$. Therefore, the nonholonomic car is flat, with $(x, y)$ as a flat output, at any $q=\left(x, y, \theta_{0}, \theta_{1}\right)$ where $\theta_{1}-\theta_{0} \neq \pm \frac{\pi}{2}$. Actually, the singularities at $\theta_{0}= \pm \frac{\pi}{2}$ are related with the domain of inversion: when calculating $\theta_{0}$ in terms of $x, y, \dot{x}=u_{1} \cos \left(\theta_{1}-\theta_{0}\right) \cos \theta_{0}$ and $\dot{y}=u_{1} \cos \left(\theta_{1}-\theta_{0}\right) \sin \theta_{0}$, we have to invert either $\tan \theta_{0}=\frac{\dot{y}}{\dot{x}}$ or $\cot \theta_{0}=\frac{\dot{x}}{\dot{y}}$.

Thirdly, and most interestingly, the nature of the singularity at $\theta_{1}-\theta_{0}= \pm \frac{\pi}{2}$ is completely different. It is an intrinsic singularity of the flat output $h=(x, y)$ (see Sect. 2.1 for details), nevertheless the nonholonomic car is flat at $\theta_{1}-\theta_{0}= \pm \frac{\pi}{2}$ if we choose another flat output! To see this, define two functions

$$
\begin{aligned}
& \psi_{1}\left(x, y, \theta_{0}\right)=x \sin \theta_{0}-y \cos \theta_{0} \\
& \psi_{2}\left(x, y, \theta_{0}\right)=x \cos \theta_{0}+y \sin \theta_{0}
\end{aligned}
$$

and consider the change of coordinates (local diffeomorphism)

$$
\begin{aligned}
& y_{1}=\theta_{0} \\
& y_{2}=\psi_{1}\left(x, y, \theta_{0}\right) \\
& y_{3}=\psi_{2}\left(x, y, \theta_{0}\right) \\
& y_{4}=\cot \left(\theta_{1}-\theta_{0}\right)-\psi_{1}\left(x, y, \theta_{0}\right)
\end{aligned}
$$

followed by the invertible feedback

$$
\begin{aligned}
& v_{1}=u_{1} \sin \left(\theta_{1}-\theta_{0}\right) \\
& v_{2}=u_{1} \sin \left(\theta_{1}-\theta_{0}\right)\left(\csc ^{2}\left(\theta_{1}-\theta_{0}\right)-\psi_{2}\left(x, y, \theta_{0}\right)\right)-u_{2} \csc ^{2}\left(\theta_{1}-\theta_{0}\right),
\end{aligned}
$$

which also brings $\Sigma_{\text {car }}$ into the chained form, but this time around $\theta_{1}-\theta_{0}= \pm \frac{\pi}{2}$,

$$
\left\{\begin{array}{l}
\dot{y}_{1}=v_{1} \\
\dot{y}_{2}=y_{3} v_{1} \\
\dot{y}_{3}=y_{4} v_{1} \\
\dot{y}_{4}=v_{2}
\end{array}\right.
$$

Thus $\left(\bar{h}_{1}, \bar{h}_{2}\right)=\left(\theta_{0}, \psi_{1}\right)=\left(\theta_{0}, x \sin \theta_{0}-y \cos \theta_{0}\right)$ is another flat output of the nonholonomic car, valid around $\theta_{1}-\theta_{0}= \pm \frac{\pi}{2}$, but singular at $\theta_{1}-\theta_{0}=0, \pm \pi$ (notice that the same singularity $v_{1}(t)=u_{1}(t)=0$, as previously, occurs in the control space).

A series of natural questions arises: are there other flat outputs of the nonholonomic car and, if so, how many and how to describe them? More generally, how to characterize all flat outputs of any 2-input driftless control system and how to describe their singular loci and singular controls? The aim of this paper is to give complete answers to those questions.

This paper is organized as follows. In Section 1, we define the crucial notion of flatness and recall a description of flat driftless 2-input systems. In Section 2, we give our main results. Namely, we characterize all flat outputs of driftless 2-input systems and give a way of parameterizing them: it turns out that all flat outputs can be 
parameterized by an arbitrary function of three intrinsically defined variables. We also construct a system of 1st order PDE's whose solutions are flat outputs of a given system. We illustrate our results by describing, in Section 3, all flat outputs of the nonholonomic car (1-trailer system) which we have just discussed and then the $n$-trailer system. We prove our results in Section 4.

\section{Flatness of DRIFtless two-INPUT CONTROL SYSTEMS}

The notion of flatness has been introduced in control theory by Fliess et al. [5-7] (see also [1,12,13,25,2729,31]), and has attracted a lot of attention because of its extensive applications in constructive controllability and trajectory tracking, compare [21] and references therein. A similar notion (of underdetermined systems of differential equations that are integrable without integration) has already been studied by Cartan [3] and Hilbert [10].

Throughout this paper, the word smooth will always mean $\mathrm{C}^{\infty}$-smooth. Consider the smooth nonlinear control system

$$
\Xi: \dot{x}=f(x, u) \text {, }
$$

where $x \in X$, an $n$-dimensional manifold, and $u \in U$, an $m$-dimensional manifold. Roughly speaking, the system $\Xi$ is said to be flat if there exist an integer $k$ and smooth functions $h_{i}=h_{i}\left(x, u, \dot{u}, \ldots, u^{(k)}\right), 1 \leq i \leq m$, (called flat outputs), as well as smooth maps $\gamma$ and $\delta$, such that the state $x$ and the control $u$ can be represented as

$$
\begin{aligned}
& x=\gamma\left(h, \dot{h}, \ldots, h^{(k)}\right) \\
& u=\delta\left(h, \dot{h}, \ldots, h^{(k)}\right),
\end{aligned}
$$

where $h=\left(h_{1}, \ldots, h_{m}\right)^{\top}$.

There are three integers (and not only $k$ ) hidden. Of course, we need less derivatives of $u$ ( $\operatorname{say,~up~to~} u^{(r)}$ ), to define $h_{i}$ than to get the representation of the state $x$ and the control $u$ (say up to $h^{(k)}$ ). Moreover, the representation (1.1) is, in general, only locally valid (in both: the state and control spaces) and we have to specify how many derivatives of $u$ (say, up to $u^{(l)}$, higher order derivatives being arbitrary) are involved in defining a neighborhood in the space of controls such that along the corresponding state trajectories the representation (1.1) holds. Those observations lead us to give the following definition.

Given any integer $l$, we associate to $\Xi$ its $l$-prolongation $\Xi^{l}$ given by

$$
\begin{aligned}
\dot{x} & =f\left(x, u^{0}\right) \\
\dot{u}^{0} & =u^{1} \\
\Xi^{l}: \quad & \vdots \\
\dot{u}^{l} & =u^{l+1},
\end{aligned}
$$

which can be considered as a control system on $X^{l}=X \times U \times \mathbb{R}^{m l}$, whose state variables are $\left(x, u^{0}, u^{1}, \ldots, u^{l}\right)$ and whose $m$ controls are the $m$ components of $u^{l+1}$. Denote $\bar{u}^{l}=\left(u^{0}, u^{1}, \ldots, u^{l}\right)$. For any integer $p \geq r$ we define by $\pi_{r}^{p}: X^{p} \rightarrow X^{r}$ the projection $\pi_{r}^{p}\left(x, u^{0}, \ldots, u^{r}, u^{r+1}, \ldots, u^{p}\right)=\left(x, u^{0}, \ldots, u^{r}\right)$.

Definition 1.1. The system $\Xi$ is flat at $\left(x_{0}, \bar{u}_{0}^{l}\right) \in X^{l}=X \times U \times \mathbb{R}^{m l}$ if there exist a neighborhood $\mathcal{O}^{l}$ of $\left(x_{0}, \bar{u}_{0}^{l}\right)$, an open subset $\mathcal{O}^{r} \subset X^{r}$, where $-1 \leq r \leq l$, satisfying $\pi_{r}^{l}\left(\mathcal{O}^{l}\right) \subset \mathcal{O}^{r}$, and $m$ smooth functions $h_{i}=h_{i}\left(x, u^{0}, u^{1}, \ldots, u^{r}\right), 1 \leq i \leq m$, called flat outputs, defined in $\mathcal{O}^{r}$ and having the following property: there exist an integer $k$ and smooth functions $\gamma_{i}, 1 \leq i \leq n$, and $\delta_{j}, 1 \leq j \leq m$, such that we have

$$
\begin{aligned}
& x_{i}=\gamma_{i}\left(h, \dot{h}, \ldots, h^{(k)}\right) \\
& u_{j}=\delta_{j}\left(h, \dot{h}, \ldots, h^{(k)}\right),
\end{aligned}
$$

where $h=\left(h_{1}, \ldots, h_{m}\right)^{\top}$, meaning that the compositions on the right hand sides are well defined in $\left(\pi_{r}^{k+r}\right)^{-1}\left(\mathcal{O}^{r}\right)$ and the equations hold along any trajectory $x(t)$ given by a control $u(t)$ that satisfy $\left(x(t), u(t), \dot{u}(t), \ldots, u^{(l)}(t)\right) \in$ $\mathcal{O}^{l}$. 
When necessary to indicate the number of derivatives on which the flat outputs $h_{i}$ depend, we will specify that the system $\Xi$ is $\left(x, u, \ldots, u^{r}\right)$-flat and in the particular case of $h_{i}=h_{i}(x)$, that is $r=-1$, we will say that the system is $x$-flat.

Our definition is equivalent to the standard "infinite dimensional" definition of flatness (based on a LieBäcklund framework $[7,13,28]$ ), which is more compact but unable to specify the constants $r, l$ and $k$ that are important in our analysis. The above definition exhibits a role of three integers involved in the notion of flatness: $r, l$ and $k$. Clearly, $r$ shows on how many derivatives of $u$ flat outputs depend while $k$ calculates the number of time-derivative of $h_{i}$ 's needed to express all components of $x$ and $u$. Finally, $l$ indicates the number of derivatives of $u$ necessary to define a neighborhood in which the desired representation of $x_{i}$ and $u_{i}$ (flatness property) is defined.

In general, the three integers can be different as shown by the following analysis of the nonholonomic car, considered in Introduction, for which we have $r=-1, l=0$ and $k=3$. Indeed, the system is $x$-flat because its flat outputs $h_{1}$ and $h_{2}$ (as well as $\bar{h}_{1}$ and $\bar{h}_{2}$ ) depend on configurations only. We have $l=0$ since the system is $x$-flat in a neighborhood $\mathcal{O}^{0}$ of any $\left(q_{0}, u_{0}^{0}\right) \in \mathbb{R}^{2} \times S^{1} \times S^{1} \times \mathbb{R}^{2}$ such that the first component $u_{10}^{0}$ of the control value $u_{0}^{0}$ satisfies $u_{10}^{0} \neq 0$. The shape of $\mathcal{O}^{0}$ depends on the point $q_{0}$ (see Introduction) and on the control value $u_{0}^{0}$ but $\mathcal{O}^{0}$ cannot be replaced by a neighborhood in the state space $\mathbb{R}^{2} \times S^{1} \times S^{1}$ only (that is, $l$ cannot be taken as minus one) because $u_{10}^{0}=0$ is an intrinsic singularity of flatness (see Thm. 1.2 below). Finally, $k=3$ because we have to use the time derivatives of order three of $h_{1}$ and $h_{2}$ in order to describe all states and controls.

In this paper, we deal only with two-input driftless (equivalently, control-linear) systems of the form

$$
\Sigma: \dot{x}=f_{1}(x) u_{1}+f_{2}(x) u_{2}
$$

on an $(n+2)$-dimensional manifold $M$, where $f_{1}$ and $f_{2}$ are $\mathrm{C}^{\infty}$-smooth vector fields independent everywhere on $M$ and $u=\left(u_{1}, u_{2}\right)^{\top} \in \mathbb{R}^{2}$. To this system, we associate the distribution $\mathcal{D}$ spanned by the vector fields $f_{1}, f_{2}$, which will be denoted by $\mathcal{D}=\operatorname{span}\left\{f_{1}, f_{2}\right\}$. Consider another two-input driftless system

$$
\tilde{\Sigma}: \dot{\tilde{x}}=\tilde{f}_{1}(\tilde{x}) \tilde{u}_{1}+\tilde{f}_{2}(\tilde{x}) \tilde{u}_{2}
$$

where $\tilde{f}_{1}$ and $\tilde{f}_{2}$ are $\mathrm{C}^{\infty}$-smooth vector fields on $\tilde{M}$. Form the matrices $f(x)=\left(f_{1}(x), f_{2}(x)\right)$ and $\tilde{f}(\tilde{x})=$ $\left(\tilde{f}_{1}(\tilde{x}), \tilde{f}_{2}(\tilde{x})\right)$. The systems $\Sigma$ and $\tilde{\Sigma}$ are feedback equivalent if there exist an invertible $(2 \times 2)$-matrix $\beta$, whose entries $\beta_{i j}, 1 \leq i, j \leq 2$, are $\mathrm{C}^{\infty}$-smooth functions on $M$, and a diffeomorphism $\Psi: M \rightarrow \tilde{M}$ such that $\mathrm{D} \Psi(x) f(x) \beta(x)=\tilde{f}(\Psi(x))$. It is easily seen that $\Sigma$ and $\tilde{\Sigma}$ are locally feedback equivalent if and only if the associated distributions $\mathcal{D}=\operatorname{span}\left\{f_{1}, f_{2}\right\}$ and $\tilde{\mathcal{D}}=\operatorname{span}\left\{\tilde{f}_{1}, \tilde{f}_{2}\right\}$ are locally equivalent via $\Psi$, i.e., $\mathrm{D} \Psi(x)(\mathcal{D}(x))=\tilde{\mathcal{D}}(\Psi(x))$.

The derived flag of a distribution $\mathcal{D}$ is the sequence of modules of vector fields $\mathcal{D}^{(0)} \subset \mathcal{D}^{(1)} \subset \cdots$ defined inductively by

$$
\mathcal{D}^{(0)}=\mathcal{D} \quad \text { and } \quad \mathcal{D}^{(i+1)}=\mathcal{D}^{(i)}+\left[\mathcal{D}^{(i)}, \mathcal{D}^{(i)}\right], \quad \text { for } \quad i \geq 0 .
$$

The Lie flag of $\mathcal{D}$ is the sequence of modules of vector fields $\mathcal{D}_{0} \subset \mathcal{D}_{1} \subset \cdots$ defined inductively by

$$
\mathcal{D}_{0}=\mathcal{D} \quad \text { and } \quad \mathcal{D}_{i+1}=\mathcal{D}_{i}+\left[\mathcal{D}_{0}, \mathcal{D}_{i}\right], \quad \text { for } \quad i \geq 0
$$

In general, the derived and Lie flags are different though for any point $x$, the inclusion $\mathcal{D}_{i}(x) \subset \mathcal{D}^{(i)}(x)$ holds, for $i \geq 0$. A characteristic vector field of a distribution $\mathcal{D}$ is a vector field $f$ that belongs to $\mathcal{D}$ and satisfies $[f, \mathcal{D}] \subset \mathcal{D}$. The characteristic distribution of $\mathcal{D}$, which will be denoted by $\mathcal{C}$, is the subdistribution spanned by all its characteristic vector fields. It follows directly from the Jacobi identity that the characteristic distribution is always involutive but, in general, it need not be of constant rank.

The problem of flatness of driftless 2-input systems has been studied and solved by Martin and Rouchon [20] (see also [19] and a related work of Cartan [3]). Their important result proves that a system is flat if and only 
if its associated distribution $\mathcal{D}$ satisfies, on an open and dense subset $M^{\prime}$ of $M$, the conditions

$$
\operatorname{rank} \mathcal{D}^{(i)}=i+2, \quad 0 \leq i \leq n
$$

A distribution $\mathcal{D}$ is called a Goursat structure (also a "système en drapeau" in [15] and a Goursat flag in [22]) if it satisfies the conditions (1.2) at any point $x \in M$. It is known since the work of von Weber [32], Cartan [3] and Goursat [9] that the conditions (1.2) imply that on an open and dense subset $M^{\prime \prime}$ of $M$, the distribution $\mathcal{D}$ can be brought into the Goursat normal form, or equivalently, the corresponding control system is locally feedback equivalent to the chained form:

$$
\Sigma_{\text {chain }}:\left\{\begin{aligned}
\dot{z}_{1} & =v_{1} \\
\dot{z}_{2} & =z_{3} v_{1} \\
\dot{z}_{3} & =z_{4} v_{1} \\
& \vdots \\
\dot{z}_{n+1} & =z_{n+2} v_{1} \\
\dot{z}_{n+2} & =v_{2} .
\end{aligned}\right.
$$

It is easy to see that $\Sigma_{\text {chain }}$ is $x$-flat with $x$-flat outputs chosen as $h=\left(h_{1}, h_{2}\right)=\left(z_{1}, z_{2}\right)$ and provided that the control $v_{1} \neq 0$ (compare Introduction, where we brought the nonholonomic car system into the chained form for $\operatorname{dim} M=4$ ). Giaro et al. [8] were the first to observe the existence of singular points in the problem of transforming a distribution of rank two into the Goursat normal form. Murray [23] proved that the feedback equivalence of $\Sigma$ to the chained form $\Sigma_{\text {chain }}$ (or, in other words, equivalence of the associated distribution to the Goursat normal form), around an arbitrary point $x_{0}$ requires, in addition to (1.2), the regularity condition (see Them. 1.2 below)

$$
\operatorname{dim} \mathcal{D}^{(i)}\left(x_{0}\right)=\operatorname{dim} \mathcal{D}_{i}\left(x_{0}\right), \quad 0 \leq i \leq n .
$$

A natural question arises: can $\Sigma$ be locally flat at a singular point of $\mathcal{D}$, i.e., at a point not satisfying the regularity condition (1.4)? In other words, can a driftless 2-input system be flat without being locally equivalent to the chained form? Theorem 1.2 answers this question (in what concerns $x$-flatness).

Let $\mathcal{D}$ be any distribution of rank two such that $\operatorname{rank} \mathcal{D}^{(1)}=3$ and $\operatorname{rank} \mathcal{D}^{(2)}=4$. Then there exists a distribution $\mathcal{C}_{1} \subset \mathcal{D}$ of corank one which is characteristic for $\mathcal{D}^{(1)}$, i.e., $\left[\mathcal{C}_{1}, \mathcal{D}^{(1)}\right] \subset \mathcal{D}^{(1)}$. Indeed, the above rank assumptions imply that (after permuting $f_{1}$ and $f_{2}$, if necessary) there exists a smooth function $\alpha$ such that $\left[f_{2},\left[f_{1}, f_{2}\right]\right]=\alpha\left[f_{1},\left[f_{1}, f_{2}\right]\right] \bmod \mathcal{D}^{(1)}$. It follows that $\left[f_{2}-\alpha f_{1},\left[f_{1}, f_{2}\right]\right]=0 \bmod \mathcal{D}^{(1)}$ and hence $\mathcal{C}_{1}=\operatorname{span}\left\{f_{2}-\alpha f_{1}\right\}$. Let $U_{\text {sing }}(x)$ be the 1-dimensional subspace of $\mathbb{R}^{2}$ such that for any feedback control $\left(u_{1}(x), u_{2}(x)\right)^{\top}=u(x) \in U_{\text {sing }}(x)$, we have $f_{1}(x) u_{1}(x)+f_{2}(x) u_{2}(x) \in \mathcal{C}_{1}(x)$ (clearly, $U_{\text {sing }}(x)$ is spanned by $\left.(\alpha(x),-1)^{\top}\right)$. Any control $u(t) \in U_{\text {sing }}(x(t))$ will be called singular and the trajectories of the system governed by a singular control remain tangent to the characteristic subdistribution $\mathcal{C}_{1}$. We have just given the definition of $U_{\text {sing }}(x)$ for $\operatorname{dim} M \geq 4$ ( $\operatorname{since}$ we have used $\operatorname{rank} \mathcal{D}^{(2)}=4$ ). If $\operatorname{dim} M=3$, we define $U_{\text {sing }}(x)=0 \in \mathbb{R}^{2}$. Note that if $l=0$, we will denote a fixed control value by $u_{0}$ (instead of more complicated $u_{0}^{0}$ ).

Theorem 1.2. Consider a 2-input driftless control system $\Sigma: \dot{x}=f_{1}(x) u_{1}+f_{2}(x) u_{2}$, where $x \in M$, an $(n+2)$-dimensional manifold, $n \geq 1$. Assume that the distribution $\mathcal{D}=\operatorname{span}\left\{f_{1}, f_{2}\right\}$ associated to $\Sigma$ is a Goursat structure, that is, satisfies $\operatorname{rank} \mathcal{D}^{(i)}=i+2$, for $0 \leq i \leq n$, everywhere on $M$. Then the following conditions are equivalent:

(i) $\Sigma$ is $x$-flat at $\left(x_{0}, \bar{u}_{0}^{l}\right) \in M \times \mathbb{R}^{2(l+1)}$, for a certain $l \geq 0$;

(ii) $\Sigma$ is $x$-flat at $\left(x_{0}, u_{0}\right) \in M \times \mathbb{R}^{2}$;

(iii) $\operatorname{dim} \mathcal{D}^{(i)}\left(x_{0}\right)=\operatorname{dim} \mathcal{D}_{i}\left(x_{0}\right)$, for $0 \leq i \leq n$, and $u_{0} \notin U_{\text {sing }}\left(x_{0}\right)$;

(iv) $\Sigma$ is locally, around $x_{0}$, feedback equivalent to the chained form $\Sigma_{\text {chain }}$ and $u_{0} \notin U_{\text {sing }}\left(x_{0}\right)$. 
We assume that $\mathcal{D}$ satisfies $\operatorname{rank} \mathcal{D}^{(i)}=i+2$, for $0 \leq i \leq n$, so the characteristic distribution $\mathcal{C}_{1}$ and the set of singular controls $U_{\text {sing }}$ are well defined. The above theorem implies that a driftless 2 -input system is never flat at $\left(x_{0}, u_{0}\right)$ such that $u_{0} \in U_{\text {sing }}\left(x_{0}\right)$. Therefore any $x$-flat outputs $\left(\varphi_{1}, \varphi_{2}\right)$ become singular in the control space (at $u_{0} \in U_{\text {sing }}$ ) but they may also exhibit singularities in the state space $M$. To formalize this, assume that a pair of functions $\left(\varphi_{1}, \varphi_{2}\right)$ defined in an open set $\mathcal{M} \subset M$ (for simplicity $\mathcal{M}$ stands for an open set $\mathcal{O}^{-1}$ ) are $x$-flat outputs at a point $\left(x_{0}, u_{0}\right) \in M \times \mathbb{R}^{2}$, that is, there exists a neighborhood $\mathcal{O}^{0} \subset M \times \mathbb{R}^{2}$, satisfying $\mathcal{O}^{0} \subset \pi^{-1}(\mathcal{M})$, where $\pi(x, u)=x$, in which the conditions of Definition 1.1 hold. Denote by $\tilde{\mathcal{M}} \subset \mathcal{M}$ the set of points such that for any $x \in \tilde{\mathcal{M}}$, the pair of functions $\left(\varphi_{1}, \varphi_{2}\right)$ is an $x$-flat output at $(x, u)$ for some control $u=u(x)$. The complement of $\tilde{\mathcal{M}}$, i.e., $\mathcal{M} \backslash \tilde{\mathcal{M}}$, will be called the singular locus of $\left(\varphi_{1}, \varphi_{2}\right)$ and denoted by $\operatorname{Sing}\left(\varphi_{1}, \varphi_{2}\right)$, that is, for any $x \in \operatorname{Sing}\left(\varphi_{1}, \varphi_{2}\right)$, the pair $\left(\varphi_{1}, \varphi_{2}\right)$ is not an $x$-flat output.

The interest of the above theorem is two-fold. First, together with its proof, it will allow us to characterize all $x$-flat outputs of driftless 2-input systems (see Sect. 2). Secondly, it shows that a Goursat structure is $x$-flat at points $x_{0}$ satisfying $\operatorname{dim} \mathcal{D}^{(i)}\left(x_{0}\right)=\operatorname{dim} \mathcal{D}_{i}\left(x_{0}\right)$, for $0 \leq i \leq n$, only, that is, at regular points of $\mathcal{D}$. Martin and Rouchon asked in [19] (see also [20]) whether a Goursat structure $\mathcal{D}$ is flat (dynamically linearizable) at points that do not satisfy $\operatorname{dim} \mathcal{D}^{(i)}\left(x_{0}\right)=\operatorname{dim} \mathcal{D}_{i}\left(x_{0}\right)$. So our result gives a negative answer to their question (for $x$-flatness). Any Goursat structure can be brought into a generalization of the Goursat normal form, called Kumpera-Ruiz normal form (see $[15,22,26]$ ). It follows that none of Kumpera-Ruiz normal forms is $x$-flat (except for the regular Kumpera-Ruiz normal form, that is, Goursat normal form). In particular, the system

$$
\left\{\begin{array}{l}
\dot{x}_{1}=x_{5} u_{1} \\
\dot{x}_{2}=x_{3} x_{5} u_{1} \\
\dot{x}_{3}=x_{4} x_{5} u_{1} \\
\dot{x}_{4}=u_{1} \\
\dot{x}_{5}=u_{2}
\end{array}\right.
$$

which is historically the first discovered Kumpera-Ruiz normal form [8], is not $x$-flat at any point of its singular locus $\left\{x \in \mathbb{R}^{5}: x=0\right\}$. This answers negatively another question of [19].

It is known (see $[14,16,22,26])$ that the model of $n$-trailer system is a Goursat structure at any configuration point but is equivalent to the chained form out of the singular locus only, that is, if all angles $\theta_{i+1}-\theta_{i}$ between two consecutive wheels are not $\pm \frac{\pi}{2}$ (except for $\theta_{1}-\theta_{0}$ which is the most far from the top of the train). Therefore our theorem implies that at any singular configuration $\theta_{i+1}-\theta_{i}= \pm \frac{\pi}{2}, i \geq 1$, the $n$-trailer system is not $x$-flat.

The proof of Theorem 1.2 is given in Section 4.2 and is based on normal forms at singular points (introduced in $[15,22,26]$ and called in the latter the Kumpera-Ruiz normal forms) and on the following result which is of independent interest (and is also proved in Sect. 4.2). It turns out that flat outputs and the chained form $\Sigma_{\text {chain }}$ are compatible: in fact, for any given pair of flat outputs $\left(\varphi_{1}, \varphi_{2}\right)$ of a system feedback equivalent to $\Sigma_{\text {chain }}$, we can bring the system, locally, to the chained form $\Sigma_{\text {chain }}$ for which $\varphi_{1}$ and $\varphi_{2}+k_{0} \varphi_{1}$, for a suitable constant $k_{0} \in \mathbb{R}$, serve as the two top variables (after permuting $\varphi_{1}$ and $\varphi_{2}$, if necessary).

To state that compatibility result precisely, observe that if $\left(\varphi_{1}, \varphi_{2}\right)$ is a flat output at $\left(x_{0}, \bar{u}_{0}^{l}\right)$, then so is $\left(\tilde{\varphi}_{1}, \tilde{\varphi}_{2}\right)$, where $\tilde{\varphi}_{i}=\varphi_{i}-l_{i}$, where $l_{i} \in \mathbb{R}$. By taking $l_{i}=\varphi_{i}\left(x_{0}\right), 1 \leq i \leq 2$, we can always assume (and we will do it throughout the paper) that the flat output satisfies $\varphi_{1}\left(x_{0}\right)=\varphi_{2}\left(x_{0}\right)=0$. We will say that local coordinates $z=\left(z_{1}, \ldots, z_{n}\right)^{\top}=\Psi(x)$ are centered at $x_{0}$ if the local diffomorphism $z=\Psi(x)$ satisfies $\Psi\left(x_{0}\right)=0$.

Proposition 1.3. Consider a driftless 2-input smooth control system $\Sigma$, defined on a manifold $M$ of dimension $n+2$, whose associated distribution $\mathcal{D}$ satisfies $\operatorname{rank} \mathcal{D}^{(i)}=\operatorname{rank} \mathcal{D}_{i}=i+2$, for $0 \leq i \leq n$. Given any pair $\left(\varphi_{1}, \varphi_{2}\right)$ of flat outputs at $\left(x_{0}, u_{0}\right) \in M \times \mathbb{R}^{2}$, there exists a feedback transformation $(\Psi, \beta)$ around $x_{0}$ bringing the system $\Sigma$ into the chained form $\Sigma_{\text {chain }}$, given by (1.3), and centered at $x_{0}$, such that $z_{1}=\varphi_{1}$ and $z_{2}=\varphi_{2}+k_{0} \varphi_{1}$ (after permuting $\varphi_{1}$ and $\varphi_{2}$, if necessary), where $k_{0} \in \mathbb{R}$ is a constant. 


\section{Characterization of flat outputs}

\subsection{Main theorems}

Recall a useful result due to Cartan [3] whose proof can be found in $[15,20,26]$.

Lemma 2.1 (Cartan). Consider a rank two distribution $\mathcal{D}$ defined on a manifold $M$ of dimension $n+2$, for $n \geq 2$. If $\mathcal{D}$ satisfies $\operatorname{rank} \mathcal{D}^{(i)}=i+2$, for $0 \leq i \leq n$, everywhere on $M$, then each distribution $\mathcal{D}^{(i)}$, for $0 \leq i \leq n-2$, contains a unique involutive subdistribution $\mathcal{C}_{i+1}$ that is characteristic for $\mathcal{D}^{(i+1)}$ and has constant corank one in $\mathcal{D}^{(i)}$.

Theorem 1.2 implies that the only Goursat structures that are $x$-flat are those equivalent to the chained form (equivalently, whose associated distribution $\mathcal{D}$ is equivalent to the Goursat normal form). For this reason, we will consider in two theorems below such distributions only. Moreover, any distribution equivalent to the Goursat normal form obviously satisfies the assumptions of Lemma 2.1 and defines the involutive distribution $\mathcal{C}_{n-1}$ that is characteristic distribution for $\mathcal{D}^{(n-1)}$ and of corank one in $\mathcal{D}^{(n-2)}$. Recall that $U_{\text {sing }}(x)$ is defined as the 1-dimensional subspace of $\mathbb{R}^{2}$ formed by all $u(x)=\left(u_{1}(x), u_{2}(x)\right)^{\top}$ such that $u_{1}(x) f_{1}(x)+u_{2}(x) f_{2}(x) \in \mathcal{C}_{1}(x)$ and $U_{\text {sing }}(x)=(0,0)$ for $n=1$. For $n=1$, i.e., $\operatorname{dim} M=3$, and any pair $\varphi=\left(\varphi_{1}, \varphi_{2}\right)$ of smooth functions we will define a 1-dimensional subspace $U_{\text {sing }}^{\varphi}(x)$ of $U=\mathbb{R}^{2}$ as $U_{\text {sing }}^{\varphi}(x)=\left\{u(x) \in \mathbb{R}^{2}: u_{1}(x) f_{1}(x)+u_{2}(x) f_{2}(x) \in\right.$ $\left.\left(\operatorname{span}\left\{\mathrm{d} \varphi_{1}, \mathrm{~d} \varphi_{2}\right\}\right)^{\perp}(x)\right\}$. Notice that for $g \in \mathcal{D}$, the condition $g\left(x_{0}\right) \notin \mathcal{C}_{n-1}\left(x_{0}\right)$ (used below) states actually that $g\left(x_{0}\right) \notin \mathcal{C}_{1}\left(x_{0}\right)$.

Theorem 2.2 (characterization of flat outputs, first version). Consider a driftless 2-input smooth control system $\Sigma$ defined on a manifold $M$ of dimension $n+2 \geq 4$ whose associated distribution $\mathcal{D}$ satisfies $\operatorname{rank} \mathcal{D}^{(i)}=$ $\operatorname{rank} \mathcal{D}_{i}=i+2$, for $0 \leq i \leq n$. Fix $x_{0} \in M$ and let $g$ be an arbitrary vector field in $\mathcal{D}$ such that $g\left(x_{0}\right) \notin \mathcal{C}_{n-1}\left(x_{0}\right)$ and let $\varphi_{1}, \varphi_{2}$ be two smooth functions defined in a neighborhood $\mathcal{M}$ of $x_{0}$. Then $\left(\varphi_{1}, \varphi_{2}\right)$ is an $x$-flat output of $\Sigma$ at $\left(x_{0}, u_{0}\right), u_{0} \notin U_{\text {sing }}\left(x_{0}\right)$, if and only if the following conditions hold:

(i) $\mathrm{d} \varphi_{1}\left(x_{0}\right) \wedge \mathrm{d} \varphi_{2}\left(x_{0}\right) \neq 0$, i.e., $\mathrm{d} \varphi_{1}$ and $\mathrm{d} \varphi_{2}$ are independent at $x_{0}$;

(ii) $L_{c} \varphi_{1} \equiv L_{c} \varphi_{2} \equiv L_{c}\left(\frac{L_{g} \varphi_{2}}{L_{g} \varphi_{1}}\right) \equiv 0$, for any $c \in \mathcal{C}_{n-1}$, where the functions $\varphi_{1}$ and $\varphi_{2}$ are ordered such that $L_{g} \varphi_{1}\left(x_{0}\right) \neq 0$ which is always possible due to item (iii) below;

(iii) $\left(L_{g} \varphi_{1}\left(x_{0}\right), L_{g} \varphi_{2}\left(x_{0}\right)\right) \neq(0,0)$;

Moreover, if a pair of functions $\left(\varphi_{1}, \varphi_{2}\right)$ satisfies (i) everywhere in $\mathcal{M}$ and forms an $x$-flat output at $(x, u)$ for any $x \in \tilde{\mathcal{M}}$ and certain $u=u(x)$, where $\tilde{\mathcal{M}}$ is open and dense in $\mathcal{M}$, then

$$
\operatorname{Sing}\left(\varphi_{1}, \varphi_{2}\right)=\left\{x \in \mathcal{M}:\left(L_{g} \varphi_{1}(x), L_{g} \varphi_{2}(x)\right)=(0,0)\right\} .
$$

Theorem 2.3 (characterization of flat outputs, second version). Consider a driftless 2-input smooth control system $\Sigma$ defined on a manifold $M$ of dimension $n+2 \geq 3$ whose associated distribution $\mathcal{D}$ satisfies $\operatorname{rank} \mathcal{D}^{(i)}=$ $\operatorname{rank} \mathcal{D}_{i}=i+2$, for $0 \leq i \leq n$. Fix $x_{0} \in M$ and let $\varphi_{1}, \varphi_{2}$ be two smooth functions defined in a neighborhood $\mathcal{M}$ of $x_{0}$. Then $\left(\varphi_{1}, \varphi_{2}\right)$ is an $x$-flat output of $\Sigma$ at $\left(x_{0}, u_{0}\right), u_{0} \notin U_{\text {sing }}\left(x_{0}\right)\left(u_{0} \notin U_{\text {sing }}^{\varphi}\left(x_{0}\right)\right.$, if $\left.n=1\right)$, if and only if the following conditions hold:

(i) ${ }^{\prime} \mathrm{d} \varphi_{1}\left(x_{0}\right) \wedge \mathrm{d} \varphi_{2}\left(x_{0}\right) \neq 0$, i.e., $\mathrm{d} \varphi_{1}$ and $\mathrm{d} \varphi_{2}$ are independent at $x_{0}$;

(ii) $^{\prime} \quad \mathcal{L}=\left(\operatorname{span}\left\{\mathrm{d} \varphi_{1}, \mathrm{~d} \varphi_{2}\right\}\right)^{\perp} \subset \mathcal{D}^{n-1}$ in $\mathcal{M} ;$

(iii) ${ }^{\prime} \mathcal{D}\left(x_{0}\right)$ is not contained in $\mathcal{L}\left(x_{0}\right)$.

Moreover, if a pair of functions $\left(\varphi_{1}, \varphi_{2}\right)$ satisfies (i) ${ }^{\prime}$ everywhere in $\mathcal{M}$ and forms an $x$-flat output at $(x, u)$ for any $x \in \tilde{\mathcal{M}}$ and certain $u=u(x)$, where $\tilde{\mathcal{M}}$ is open and dense in $\mathcal{M}$, then

$$
\operatorname{Sing}\left(\varphi_{1}, \varphi_{2}\right)=\{x \in \mathcal{M}: \mathcal{D}(x) \subset \mathcal{L}(x)\} .
$$

Remark 2.4. Notice that Theorem 2.3 is valid for any $n \geq 1$ (i.e., $\operatorname{dim} M \geq 3$ ) while Theorem 2.2 is true for $n \geq 2$ only (i.e., $\operatorname{dim} M \geq 4$ ). In fact, in Theorem 2.2 we use the characteristic distribution $\mathcal{C}_{n-1}$ of $\mathcal{D}^{(n-1)}$ 
but if $\operatorname{dim} M=3$, such a distribution does not exist and therefore Theorem 2.2 can not be applied in that case. Observe that if $\operatorname{dim} M=3$, then (iii) ${ }^{\prime}$ is satisfied automatically so for any $\left(\varphi_{1}, \varphi_{2}\right)$ satisfying (i) ${ }^{\prime}$ and (ii $^{\prime}$, the singular locus $\operatorname{Sing}\left(\varphi_{1}, \varphi_{2}\right)$ in the state space $M$ is empty. On the other hand, for $\operatorname{dim} M=3$, each flat output $\varphi=\left(\varphi_{1}, \varphi_{2}\right)$ defines its singular control $U_{\text {sing }}^{\varphi}(x)$ for which $\varphi$ fails to be a flat output (although any 3 -dimensional system is flat for any $u_{0} \neq(0,0)$, as stated in Theorem 1.2-(iii)).

Remark 2.5. The two items (iii) and (iii)' are equivalent (which will be shown in the proofs of the two theorems) under the condition $\operatorname{rank} \mathcal{D}^{(i)}=\operatorname{rank} \mathcal{D}_{i}=i+2$, for $0 \leq i \leq n$, and thus the two ways of describing the singular locus of an $x$-flat output $\left(\varphi_{1}, \varphi_{2}\right)$ coincide: $\left\{x \in \mathcal{M}:\left(L_{g} \varphi_{1}(x), L_{g} \varphi_{2}(x)\right)=(0,0)\right\}=\{x \in \mathcal{M}: \mathcal{D}(x) \subset \mathcal{L}(x)\}$.

Remark 2.6. The conditions of both theorems are verifiable, i.e., given a pair of functions $\left(\varphi_{1}, \varphi_{2}\right)$ in a neighborhood of a point $x_{0}$, we can easily verify whether $\left(\varphi_{1}, \varphi_{2}\right)$ forms an $x$-flat output of a control system under considerations and verification involves derivations and algebraic operations only (without solving PDE's or bringing the system into a normal form). Moreover, the theorems allow us to find the singular locus of a given flat output $\left(\varphi_{1}, \varphi_{2}\right)$.

A natural question to ask is if there exists a lot of pairs $\left(\varphi_{1}, \varphi_{2}\right)$ which satisfy the conditions of Theorem 2.2 or 2.3? In other words, is there a lot of pairs $\left(\varphi_{1}, \varphi_{2}\right)$ which are $x$-flat outputs for a 2-input driftless control system? This question has an elegant answer given by the following theorem. Recall that $\mathcal{C}_{n-1}$ denotes the characteristic distribution of $\mathcal{D}^{(n-1)}$.

Theorem 2.7 (non-uniqueness and parameterization of $x$-flat outputs). Consider a driftless 2-input smooth control system $\Sigma$ whose associated distribution $\mathcal{D}$ satisfies $\operatorname{rank} \mathcal{D}^{(i)}=\operatorname{rank} \mathcal{D}_{i}=i+2$, for $0 \leq i \leq n$, locally around a point $x_{0} \in M$, an $(n+2)$-dimensional manifold. Let $g$ be an arbitrary vector field in $\mathcal{D}$ such that $g\left(x_{0}\right) \notin \mathcal{C}_{n-1}\left(x_{0}\right)$. Then for a given arbitrary smooth function $\varphi_{1}$ such that $L_{c} \varphi_{1}=0$, for any $c \in \mathcal{C}_{n-1}$, and $L_{g} \varphi_{1}\left(x_{0}\right) \neq 0$, there always exists a function $\varphi_{2}$ such that $\left(\varphi_{1}, \varphi_{2}\right)$ is an $x$-flat output of $\Sigma$ at $\left(x_{0}, u_{0}\right)$, $u_{0} \notin U_{\text {sing }}\left(x_{0}\right)$. Moreover, if for a given function $\varphi_{1}$ as above, the pairs $\left(\varphi_{1}, \varphi_{2}\right)$ and $\left(\varphi_{1}, \tilde{\varphi}_{2}\right)$ are both $x$-flat outputs of $\Sigma$ at $\left(x_{0}, u_{0}\right)$, then $\operatorname{span}\left\{\mathrm{d} \varphi_{1}, \mathrm{~d} \varphi_{2}\right\}(x)=\operatorname{span}\left\{\mathrm{d} \varphi_{1}, \mathrm{~d} \tilde{\varphi}_{2}\right\}(x)$, for any $x$ in a neighborhood of $x_{0}$.

Remark 2.8. Observe that $x$-flat outputs $\left(h_{1}, \ldots, h_{m}\right)$ and $\left(\tilde{h}_{1}, \ldots, \tilde{h}_{m}\right)$ of a system with $m$ controls such that $\operatorname{span}\left\{\mathrm{d} h_{1}, \ldots, \mathrm{d} h_{m}\right\}=\operatorname{span}\left\{\mathrm{d} \tilde{h}_{1}, \ldots, \mathrm{d} \tilde{h}_{m}\right\}$ can be considered as statically equivalent. Indeed, in that case there exist smooth functions $H_{i}$ and $\tilde{H}_{i}$ of $m$ variables such that $h_{i}=H_{i}\left(\tilde{h}_{1}, \ldots, \tilde{h}_{m}\right)$ and $\tilde{h}_{i}=\tilde{H}_{i}\left(h_{1}, \ldots, h_{m}\right)$. It thus follows from Theorem 2.7 that for a given arbitrary $\varphi_{1}$ (satisfying the assumptions of the theorem), the choice of $\varphi_{2}$ is unique in the sense that all functions $\varphi_{2}$ giving $x$-flat outputs $\left(\varphi_{1}, \varphi_{2}\right)$ yield, actually, statically equivalent $x$-flat outputs.

According to Theorem 2.7 and Remark 2.8 there are as many $x$-flat outputs as functions $\varphi_{1}$ satisfying $L_{c} \varphi_{1} \equiv 0$, for any $c \in \mathcal{C}_{n-1}$, and $\left(L_{g} \varphi_{1}\right)\left(x_{0}\right) \neq 0$, that is, as many as functions of well chosen three variables. In fact, the distribution $\mathcal{C}_{n-1}$ is involutive and of corank three in $T M$ and therefore any $\varphi_{1}$ is of the form $\varphi_{1}=\Phi\left(\psi_{1}, \psi_{2}, \psi_{3}\right)$ where $\Phi$ is any function of three variables and $\psi_{1}, \psi_{2}, \psi_{3}$ are arbitrary independent functions whose differentials $\mathrm{d} \psi_{i}, 1 \leq i \leq 3$, annihilate $\mathcal{C}_{n-1}$. The regularity condition $\left(L_{g} \varphi_{1}\right)\left(x_{0}\right) \neq 0$ assures that $\mathrm{d} \varphi_{1}\left(x_{0}\right)$ does not annihilate $\mathcal{D}\left(x_{0}\right)$.

Let Flat $\left(\varphi_{1}\right)$ be the codistribution spanned, around $x_{0}$, by the differentials of all $x$-flat outputs at $x_{0}$ determined by a function $\varphi_{1}$, i.e., Flat $\left(\varphi_{1}\right)=\operatorname{span}\left\{\mathrm{d} \varphi_{1}, \mathrm{~d} \varphi_{2}\right\}$, where $\varphi_{2}$ is any function such that $\left(\varphi_{1}, \varphi_{2}\right)$ is an $x$-flat output. Clearly, Flat $\left(\varphi_{1}\right)$ is well defined because of Theorem 2.7.

Corollary 2.9. If Flat $\left(\varphi_{1}\right)=\operatorname{span}\left\{\mathrm{d} \varphi_{1}, \mathrm{~d} \varphi_{2}\right\}$, then Flat $\left(\varphi_{1}\right)=\operatorname{Flat}\left(\varphi_{2}\right)$, locally around $x_{0}$.

This corollary is easy to prove and here we omit its proof.

\subsection{Finding $x$-flat outputs}

The importance of Theorem 2.2 is that it not only allows to check whether a given pair of functions forms an $x$-flat output but also, together with Theorem 2.7, to express explicitly a system of 1st order PDE's to be solved in order to calculate all $x$-flat outputs for a given 2-input driftless system. 
To this aim, choose $n-1$ vector fields $c_{1}, \ldots, c_{n-1}$ spanning the characteristic distribution $\mathcal{C}_{n-1}$ of $\mathcal{D}^{(n-1)}$. Recall that $\mathcal{C}_{n-1}$ can be easily calculated as (see, e.g., [2])

$$
\left.\mathcal{C}_{n-1}=\left\{f \in \mathcal{D}^{(n-1)}: f\right\lrcorner \mathrm{d} \omega \in\left(\mathcal{D}^{(n-1)}\right)^{\perp}\right\},
$$

where $\omega$ is any non-zero differential 1 -form annihilating $\mathcal{D}^{(n-1)}$. Fix a vector field $g$ of $\mathcal{D}$ such that $g\left(x_{0}\right) \notin$ $\mathcal{C}_{n-1}\left(x_{0}\right)$. According to Theorems 2.2 and 2.7, in order to find $\varphi_{1}$, we have to solve the following system of 1 st order PDE's

$$
\begin{aligned}
L_{c_{i}} \varphi_{1} & =0, \quad 1 \leq i \leq n-1, \\
L_{g} \varphi_{1}\left(x_{0}\right) & \neq 0 .
\end{aligned}
$$

The above system possesses solutions ( $\operatorname{since} \mathcal{C}_{n-1}$ is involutive) and the space of solutions is that of functions of three variables (since $\operatorname{corank}\left(\mathcal{C}_{n-1} \subset T M\right)=3$ ).

Now we will establish a system of equations for $\varphi_{2}$. According to Theorem 2.2, it is given by

$$
\begin{aligned}
& L_{c_{i}} \varphi_{2}=0, \quad 1 \leq i \leq n-1 \\
& L_{c_{i}}\left(\frac{L_{g} \varphi_{2}}{L_{g} \varphi_{1}}\right)=0, \quad 1 \leq i \leq n-1 .
\end{aligned}
$$

The last $n-1$ equations are equivalent to $\left(L_{g} \varphi_{1}\right) L_{c_{i}} L_{g} \varphi_{2}-\left(L_{g} \varphi_{2}\right) L_{c_{i}} L_{g} \varphi_{1}=0,1 \leq i \leq n-1$. Applying $L_{\left[c_{i}, g\right]} \psi=L_{c_{i}} L_{g} \psi-L_{g} L_{c_{i}} \psi$ and taking into account that $L_{c_{i}} \varphi_{1}=L_{c_{i}} \varphi_{2}=0$, we get

$$
\left(L_{g} \varphi_{1}\right) L_{\left[c_{i}, g\right]} \varphi_{2}-\left(L_{\left[c_{i}, g\right]} \varphi_{1}\right) L_{g} \varphi_{2}=0, \quad 1 \leq i \leq n-1,
$$

which we rewrite as

$$
L_{v_{i}} \varphi_{2}=0, \quad 1 \leq i \leq n-1,
$$

where the vector fields $v_{1}, \ldots, v_{n-1}$ are given by $v_{i}=\left(L_{g} \varphi_{1}\right)\left[c_{i}, g\right]-\left(L_{\left[c_{i}, g\right]} \varphi_{1}\right) g, \quad 1 \leq i \leq n-1$. We want to emphasize that the vector fields $v_{i}$ are easily calculable in terms of the vector fields $g, c_{1}, \ldots, c_{n-1}$ and the chosen solution $\varphi_{1}$. So finally, we have to solve the system $L_{c_{i}} \varphi_{2}=L_{v_{i}} \varphi_{2}=0$ which, surprisingly, consists of $2(n-1)$ 1st order PDE's on an $(n+2)$-dimensional manifold. We will show below that this system reduces, actually, to $n$ equations.

\subsection{Reducing equations for the flat output second component}

By Cartan's Lemma 2.1 we have $\mathcal{C}_{1} \subset \mathcal{C}_{2} \subset \cdots \subset \mathcal{C}_{n-1}$ and $\operatorname{corank}\left(\mathcal{C}_{i} \subset \mathcal{D}^{(i-1)}\right)=1$, for $1 \leq i \leq$ $n-1$. Thus we can always choose vector fields $c_{1}, \ldots, c_{n-1}$ such that $\mathcal{C}_{n-1}=\operatorname{span}\left\{c_{1}, \ldots, c_{n-1}\right\}$ and $\mathcal{C}_{n-2}=$ $\operatorname{span}\left\{c_{1}, \ldots, c_{n-2}\right\}$. and hence $c_{n-1}\left(x_{0}\right) \notin \mathcal{C}_{n-2}\left(x_{0}\right)$. Fix a vector field $g \in \mathcal{D}$ such that $g\left(x_{0}\right) \notin \mathcal{C}_{n-1}\left(x_{0}\right)$ and clearly we have $\mathcal{D}^{(n-2)}=\operatorname{span}\left\{c_{1}, \ldots, c_{n-2}, c_{n-1}, g\right\}$. We claim that the system

$$
\begin{aligned}
& L_{c_{i}} \varphi_{2}=0, \quad 1 \leq i \leq n-1 \\
& L_{v_{i}} \varphi_{2}=0, \quad 1 \leq i \leq n-1,
\end{aligned}
$$

where $v_{i}=\left(L_{g} \varphi_{1}\right)\left[c_{i}, g\right]-\left(L_{\left[c_{i}, g\right]} \varphi_{1}\right) g$, can be reduced to a system of $n$ equations. Since $\mathcal{C}_{n-2}$ is the characteristic distribution of $\mathcal{D}^{(n-2)}$, we get

$$
\left[c_{i}, g\right]=\beta_{i} g \bmod \mathcal{C}_{n-1}, 1 \leq i \leq n-2,
$$

where $\beta_{i}$, for $1 \leq i \leq n-2$, are smooth functions defined in a neighborhood of $x_{0}$. Equation (2.6) implies that (recall that $L_{c} \varphi_{1}=0$, for any $c \in \mathcal{C}_{n-1}$ )

$$
L_{\left[c_{i}, g\right]} \varphi_{1}=L_{\beta_{i} g} \varphi_{1}=\beta_{i} L_{g} \varphi_{1} .
$$


Therefore, for $1 \leq i \leq n-2$,

$$
\begin{aligned}
v_{i} & =\left(L_{g} \varphi_{1}\right)\left[c_{i}, g\right]-\left(L_{\left[c_{i}, g\right]} \varphi_{1}\right) g \\
& =\left(L_{g} \varphi_{1}\right) \beta_{i} g-\beta_{i}\left(L_{g} \varphi_{1}\right) g \bmod \mathcal{C}_{n-1} \\
& =0 \bmod \mathcal{C}_{n-1}
\end{aligned}
$$

It follows that the equations $L_{c_{i}} \varphi_{2}=0$, for $c_{i} \in \mathcal{C}_{n-1}$, imply $L_{v_{i}} \varphi_{2}=0,1 \leq i \leq n-2$, and therefore the system $(2.5)$ is equivalent to the following system of $n$ equations

$$
\begin{aligned}
L_{c_{i}} \varphi_{2} & =0, \quad 1 \leq i \leq n-1 \\
L_{v_{n-1}} \varphi_{2} & =0
\end{aligned}
$$

where $v_{n-1}=\left(L_{g} \varphi_{1}\right)\left[c_{n-1}, g\right]-\left(L_{\left[c_{n-1}, g\right]} \varphi_{1}\right) g$. Notice that $\varphi_{1}$ solves the system (2.8). We are thus looking for a solution $\varphi_{2}$ of (2.8), independent with $\varphi_{1}$, and by the Frobenious theorem the system (2.8) possesses two independent solutions if and only if the distribution $\mathcal{L}=\operatorname{span}\left\{c_{1}, \ldots, c_{n-1}, v_{n-1}\right\}=\mathcal{C}_{n-1} \oplus \operatorname{span}\left\{v_{n-1}\right\}$ is involutive.

Below we will show that $\mathcal{L}$ is, indeed, involutive and to this end it is sufficient to show that $\left[c_{i}, v_{n-1}\right] \in \mathcal{L}$, for any $1 \leq i \leq n-1$. Since $\mathcal{D}^{(n-2)}=\operatorname{span}\left\{c_{1}, \ldots, c_{n-1}, g\right\}$, we have $\mathcal{D}^{(n-1)}=\mathcal{D}^{(n-2)}+\left[\mathcal{D}^{(n-2)}, \mathcal{D}^{(n-2)}\right]=$ $\operatorname{span}\left\{c_{1}, \ldots, c_{n-1}, g,\left[c_{n-1}, g\right]\right\}$. The fact that $\mathcal{C}_{n-1}=\operatorname{span}\left\{c_{1}, \ldots, c_{n-1}\right\}$ is the characteristic distribution of $\mathcal{D}^{(n-1)}$ implies that

$$
\left[c_{i},\left[c_{n-1}, g\right]\right]=\xi_{i} g+\tau_{i}\left[c_{n-1}, g\right] \bmod \mathcal{C}_{n-1}, 1 \leq i \leq n-1,
$$

where $\xi_{i}, \tau_{i}$ are smooth functions defined in a neighborhood of $x_{0}$, and thus

$$
L_{\left[c_{i},\left[c_{n-1}, g\right]\right]} \varphi_{1}=\xi_{i} L_{g} \varphi_{1}+\tau_{i} L_{\left[c_{n-1}, g\right]} \varphi_{1}, 1 \leq i \leq n-1
$$

Observing that

$$
\begin{aligned}
L_{\left[c_{i}, g\right]} \varphi_{1} & =L_{c_{i}} L_{g} \varphi_{1}-L_{g} L_{c_{i}} \varphi_{1}=L_{c_{i}} L_{g} \varphi_{1} \\
L_{\left[c_{i},\left[c_{n-1}, g\right]\right]} \varphi_{1} & =L_{c_{i}} L_{\left[c_{n-1}, g\right]} \varphi_{1}-L_{\left[c_{n-1}, g\right]} L_{c_{i}} \varphi_{1}=L_{c_{i}} L_{\left[c_{n-1}, g\right]} \varphi_{1}
\end{aligned}
$$

and applying the relations (2.6)-(2.10), we have, for $1 \leq i \leq n-2$,

$$
\begin{aligned}
{\left[c_{i}, v_{n-1}\right]=} & {\left[c_{i},\left(L_{g} \varphi_{1}\right)\left[c_{n-1}, g\right]\right]-\left[c_{i},\left(L_{\left[c_{n-1}, g\right]} \varphi_{1}\right) g\right] } \\
= & \left(L_{g} \varphi_{1}\right)\left[c_{i},\left[c_{n-1}, g\right]\right]+\left(L_{c_{i}} L_{g} \varphi_{1}\right)\left[c_{n-1}, g\right]-\left(L_{\left[c_{n-1}, g\right]} \varphi_{1}\right)\left[c_{i}, g\right]-\left(L_{c_{i}} L_{\left[c_{n-1}, g\right]} \varphi_{1}\right) g \\
= & L_{g} \varphi_{1}\left(\xi_{i} g+\tau_{i}\left[c_{n-1}, g\right]\right)+\beta_{i}\left(L_{g} \varphi_{1}\right)\left[c_{n-1}, g\right] \\
& -\left(L_{\left[c_{n-1}, g\right]} \varphi_{1}\right) \beta_{i} g-\left(\xi_{i}\left(L_{g} \varphi_{1}\right) g+\tau_{i}\left(L_{\left[c_{n-1}, g\right]} \varphi_{1}\right) g\right) \bmod \mathcal{C}_{n-1} \\
= & \left(\tau_{i}+\beta_{i}\right) v_{n-1} \bmod \mathcal{C}_{n-1}
\end{aligned}
$$


which implies that $\left[c_{i}, v_{n-1}\right] \in \mathcal{L}$, for $1 \leq i \leq n-2$. Now consider the case $i=n-1$, that is, the Lie bracket $\left[c_{n-1}, v_{n-1}\right]$. Applying (2.9) and (2.10) for $i=n-1$, we get

$$
\begin{aligned}
{\left[c_{n-1}, v_{n-1}\right]=} & {\left[c_{n-1},\left(L_{g} \varphi_{1}\right)\left[c_{n-1}, g\right]\right]-\left[c_{n-1},\left(L_{\left[c_{n-1}, g\right]} \varphi_{1}\right) g\right] } \\
= & \left(L_{g} \varphi_{1}\right)\left[c_{n-1},\left[c_{n-1}, g\right]\right]+\left(L_{c_{n-1}} L_{g} \varphi_{1}\right)\left[c_{n-1}, g\right] \\
& -\left(L_{\left[c_{n-1}, g\right]} \varphi_{1}\right)\left[c_{n-1}, g\right]-\left(L_{c_{n-1}} L_{\left[c_{n-1}, g\right]} \varphi_{1}\right) g \\
= & \left(L_{g} \varphi_{1}\right)\left[c_{n-1},\left[c_{n-1}, g\right]\right]-\left(L_{c_{n-1}} L_{\left[c_{n-1}, g\right]} \varphi_{1}\right) g \\
= & \xi_{n-1}\left(L_{g} \varphi_{1}\right) g+\tau_{n-1}\left(L_{g} \varphi_{1}\right)\left[c_{n-1}, g\right] \\
& -\left(\xi_{n-1}\left(L_{g} \varphi_{1}\right) g+\tau_{n-1}\left(L_{\left[c_{n-1}, g\right]} \varphi_{1}\right) g\right) \bmod \mathcal{C}_{n-1} \\
= & \tau_{n-1} v_{n-1} \bmod \mathcal{C}_{n-1},
\end{aligned}
$$

which implies that $\left[c_{n-1}, v_{n-1}\right] \in \mathcal{L}$. In conclusion, the distribution $\mathcal{L}$ is involutive and hence the system (2.8) is solvable. Together with the analysis of Section 2.2 , we get the following theorem.

Theorem 2.10. Assume that a driftless two-input control system $\Sigma$, defined on an $(n+2)$-dimensional manifold $M$, is $x$-flat at $\left(x_{0}, u_{0}\right), u_{0} \notin U_{\text {sing }}\left(x_{0}\right)$, that is, the associated distribution $\mathcal{D}$ is, locally at $x_{0}$, equivalent to the Goursat normal form. Let $\mathcal{C}_{n-1}=\operatorname{span}\left\{c_{1}, \ldots, c_{n-1}\right\}$ be the characteristic distribution of $\mathcal{D}^{(n-1)}$ such that $c_{n-1}\left(x_{0}\right) \notin \mathcal{C}_{n-2}\left(x_{0}\right)$ and $g$ any vector field in $\mathcal{D}$ such that $g\left(x_{0}\right) \notin \mathcal{C}_{n-1}\left(x_{0}\right)$. Then

(i) For any smooth function $\varphi_{1}$ such that

$$
\begin{aligned}
& \text { (Flat 1) } \quad L_{c_{i}} \varphi_{1}=0, \quad 1 \leq i \leq n-1 \\
& L_{g} \varphi_{1}\left(x_{0}\right) \neq 0 \text {, }
\end{aligned}
$$

the distribution $\mathcal{L}=\operatorname{span}\left\{c_{1}, \ldots, c_{n-1}, v\right\}$ is involutive, where $v=\left(L_{g} \varphi_{1}\right)\left[c_{n-1}, g\right]-\left(L_{\left[c_{n-1}, g\right]} \varphi_{1}\right) g$.

(ii) A pair of functions $\left(\varphi_{1}, \varphi_{2}\right)$ forms an $x$-flat output of $\Sigma$ at $\left(x_{0}, u_{0}\right), u_{0} \notin U_{\text {sing }}\left(x_{0}\right)$, if and only if after a permutation (if necessary) $\varphi_{1}$ satisfies (Flat 1 ), $\mathrm{d} \varphi_{1}\left(x_{0}\right) \wedge \mathrm{d} \varphi_{2}\left(x_{0}\right) \neq 0$, and $\varphi_{2}$ satisfies

(Flat 2)

$$
\begin{aligned}
& L_{c_{i} \varphi_{2}}=0, \quad 1 \leq i \leq n-1 \\
& L_{v} \varphi_{2}=0
\end{aligned}
$$

Remark 2.11. In (ii) only one implication may need permuting $\varphi_{1}$ and $\varphi_{2}$. Indeed, if $\left(\varphi_{1}, \varphi_{2}\right)$ satisfies (Flat 1$)$ and (Flat 2), then it is an $x$-flat output (and no permutation is needed). If $\left(\varphi_{1}, \varphi_{2}\right)$ is an $x$-flat output, then at least one $\varphi_{i}, 1 \leq i \leq 2$, satisfies $L_{g} \varphi_{i}\left(x_{0}\right) \neq 0$ and we choose $\varphi_{1}$ such that $L_{g} \varphi_{1}\left(x_{0}\right) \neq 0$.

Example 2.12. To illustrate the above-presented procedure of finding flat outputs, we will consider the case of 2-input system $\Sigma: \dot{x}=f_{1}(x) u_{1}+f_{2}(x) u_{2}$ on a 4-dimensional manifold $M$. Assume that the system is $x$-flat, that is, the associated distributions $\mathcal{D}=\operatorname{span}\left\{f_{1}, f_{2}\right\}$ satisfies the conditions of Theorem 2.10. Choose a vector field $c \in \mathcal{C}_{1}$ characteristic for $\mathcal{D}^{(1)}$ and $g \in \mathcal{D}$ such that $g\left(x_{0}\right) \wedge c\left(x_{0}\right) \neq 0$. According to the above procedure we take as $\varphi_{1}$ an arbitrary solution of

$$
\begin{array}{r}
L_{c} \varphi_{1}=0 \\
L_{g} \varphi_{1}\left(x_{0}\right) \neq 0
\end{array}
$$

and, in order to find $\varphi_{2}$, we have to solve

$$
\begin{aligned}
& L_{c} \varphi_{2}=0 \\
& L_{v} \varphi_{2}=0
\end{aligned}
$$


where $v=\left(L_{g} \varphi_{1}\right)[c, g]-\left(L_{[c, g]} \varphi_{1}\right) g$. Notice that the above system of three 1st order PDE's contains a fourth one; indeed, we have $L_{v} \varphi_{1}=\left(L_{g} \varphi_{1}\right) L_{[c, g]} \varphi_{1}-\left(L_{[c, g]} \varphi_{1}\right) L_{g} \varphi_{1}=0$. The system

$$
L_{c} \varphi_{i}=L_{v} \varphi_{i}=0, \quad 1 \leq i \leq 2,
$$

admits two independent functions $\varphi_{1}$ and $\varphi_{2}$ as solutions if and only if the distribution span $\{c, v\}$ is integrable. A direct calculation shows that this is the case. All becomes clear: the involutive distribution $\operatorname{span}\{c, v\}$ is just the distribution $\mathcal{L}$ of Theorem 2.3 while $\varphi_{1}$ and $\varphi_{2}$ satisfying (2.12) are $x$-flat outputs since their differentials span the codistribution $\mathcal{L}^{\perp}$. We also see that $\mathcal{L}$ is not unique: different choices of $\varphi_{1}$ lead to different vector fields $v$ which, in turn, give different distributions $\mathcal{L}=\operatorname{span}\{c, v\}$, although all of them are involutive and thus define (via $\operatorname{span}\left\{\mathrm{d} \varphi_{1}, \mathrm{~d} \varphi_{2}\right\}=\mathcal{L}^{\perp}$ ) non equivalent flat outputs. This is in a perfect accordance with Theorem 2.7.

\section{Applications}

\subsection{A complete description of $x$-flat outputs for the nonholonomic car system}

Come back to the example of the nonholonomic car $\Sigma_{\text {car }}$ that we analyzed in Introduction:

$$
\Sigma_{\text {car }}:\left(\begin{array}{c}
\dot{x} \\
\dot{y} \\
\dot{\theta}_{0} \\
\dot{\theta_{1}}
\end{array}\right)=\left(\begin{array}{c}
\cos \left(\theta_{1}-\theta_{0}\right) \cos \theta_{0} \\
\cos \left(\theta_{1}-\theta_{0}\right) \sin \theta_{0} \\
\sin \left(\theta_{1}-\theta_{0}\right) \\
0
\end{array}\right) u_{1}+\left(\begin{array}{l}
0 \\
0 \\
0 \\
1
\end{array}\right) u_{2} .
$$

We have a characteristic vector field $c=\frac{\partial}{\partial \theta_{1}}$ and take

$$
g=\cos \left(\theta_{1}-\theta_{0}\right) \cos \theta_{0} \frac{\partial}{\partial x}+\cos \left(\theta_{1}-\theta_{0}\right) \sin \theta_{0} \frac{\partial}{\partial y}+\sin \left(\theta_{1}-\theta_{0}\right) \frac{\partial}{\partial \theta_{0}}
$$

As a first $x$-flat output we can take any function $\varphi_{1}$ satisfying $L_{c} \varphi_{1}=\frac{\partial \varphi_{1}}{\partial \theta_{1}} \equiv 0$ and $L_{g} \varphi_{1}(q) \neq 0$, that is any function $\varphi_{1}=\varphi_{1}\left(x, y, \theta_{0}\right)$ such that $L_{g} \varphi_{1}(q) \neq 0$. Let us choose one such $\varphi_{1}$ then $\varphi_{2}$ satisfies $L_{c} \varphi_{2}=L_{v} \varphi_{2}=0$, where the vector field $v$ is given by

$$
\begin{aligned}
v & =\left(L_{g} \varphi_{1}\right)[c, g]-\left(L_{[c, g]} \varphi_{1}\right) g \\
& =-\frac{\partial \varphi_{1}}{\partial \theta_{0}} \cos \theta_{0} \frac{\partial}{\partial x}-\frac{\partial \varphi_{1}}{\partial \theta_{0}} \sin \theta_{0} \frac{\partial}{\partial y}+\left(\frac{\partial \varphi_{1}}{\partial x} \cos \theta_{0}+\frac{\partial \varphi_{1}}{\partial y} \sin \theta_{0}\right) \frac{\partial}{\partial \theta_{0}} .
\end{aligned}
$$

Therefore $\varphi_{2}$ can be taken as any function $\varphi_{2}\left(x, y, \theta_{0}\right)$ satisfying $L_{v} \varphi_{2}=0$ and $\left(\mathrm{d} \varphi_{1} \wedge \mathrm{d} \varphi_{2}\right)(q) \neq 0$. Given $\varphi_{1}$ as above, the space of solutions for $\varphi_{2}$ is thus parameterized by one function of two variables but any two solutions $\varphi_{2}$ and $\tilde{\varphi}_{2}$ give statically equivalent flat outputs, that is $\operatorname{span}\left\{\mathrm{d} \varphi_{1}, \mathrm{~d} \varphi_{2}\right\}=\operatorname{span}\left\{\mathrm{d} \varphi_{1}, \mathrm{~d} \tilde{\varphi}_{2}\right\}$. On the other hand, different choices of $\varphi_{1}$ will lead to nonequivalent pairs $\left(\varphi_{1}, \varphi_{2}\right)$ of $x$-flat outputs.

To illustrate this, take $\varphi_{1}=x$, then $v=\cos \theta_{0} \frac{\partial}{\partial \theta_{0}}$ and $L_{c} \varphi_{2}=L_{v} \varphi_{2}=0$ imply that $\varphi_{2}$ is any function of the form $\varphi_{2}=\varphi_{2}(x, y)$ satisfying $\frac{\partial \varphi_{2}}{\partial y}(q) \neq 0$ (because of $\left.\left(\mathrm{d} \varphi_{1} \wedge \mathrm{d} \varphi_{2}\right)(q) \neq 0\right)$. All such functions satisfy $\operatorname{span}\left\{\mathrm{d} x, \mathrm{~d} \varphi_{2}\right\}=\operatorname{span}\left\{\mathrm{d} x, \mathrm{~d} \tilde{\varphi}_{2}\right\}$ and we can take, for instance, $\varphi_{2}=y$. This gives the well-known flat output $(x, y)$.

To see another choice, take $\varphi_{1}=\theta_{0}$, then $v=-\cos \theta_{0} \frac{\partial}{\partial x}-\sin \theta_{0} \frac{\partial}{\partial y}$ and the general solution of $L_{c} \varphi_{2}=$ $L_{v} \varphi_{2}=0$ is $\varphi_{2}=\varphi_{2}\left(\theta_{0}, x \sin \theta_{0}-y \cos \theta_{0}\right)$, which gives as an $x$-flat output $\left(\theta_{0}, x \sin \theta_{0}-y \cos \theta_{0}\right)$, the second flat output of the car system (see Introduction). Notice that the singular loci of the two choices of $x$-flat outputs are different. In fact, $\operatorname{Sing}(x, y)=\left\{\theta_{1}-\theta_{0}= \pm \frac{\pi}{2}\right\}$ and $\operatorname{Sing}\left(\theta_{0}, x \sin \theta_{0}-y \cos \theta_{0}\right)=\left\{\theta_{1}-\theta_{0}=0, \pm \pi\right\}$. 


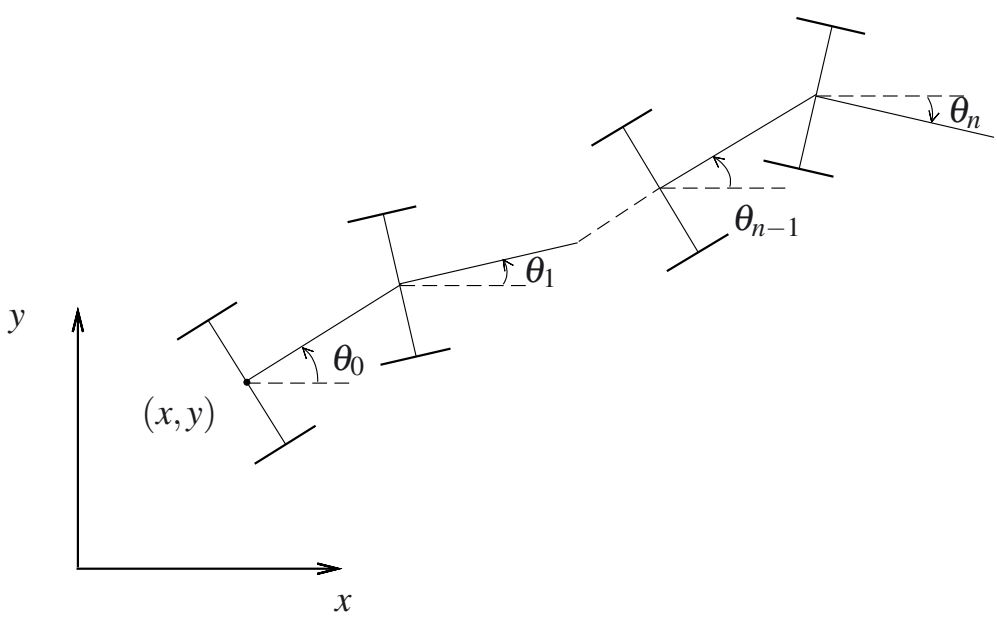

Figure 2. The $n$-trailer system.

Now take $\varphi_{1}=x+\theta_{0}$ around $\cos \theta_{0} \neq 0$, then $v=-\cos \theta_{0} \frac{\partial}{\partial x}-\sin \theta_{0} \frac{\partial}{\partial y}+\cos \theta_{0} \frac{\partial}{\partial \theta_{0}}$. Thus the general solution of $L_{c} \varphi_{2}=L_{v} \varphi_{2}=0$ is $\left.\varphi_{2}=\varphi_{2}\left(x+\theta_{0}, y-\ln \left|\cos \theta_{0}\right|\right)\right)$. We can take, for instance, $\varphi_{2}=y-\ln \left|\cos \theta_{0}\right|$ which gives a third $x$-flat output $\left(x+\theta_{0}, y-\ln \left|\cos \theta_{0}\right|\right)$ of $\Sigma_{\text {car }}$ and its singular locus is defined by

$$
\operatorname{Sing}\left(x+\theta_{0}, y-\ln \left|\cos \theta_{0}\right|\right)=\left\{\cos \theta_{0}=0\right\} \cup\left\{\cos \left(\theta_{1}-\theta_{0}\right) \cos \theta_{0}+\sin \left(\theta_{1}-\theta_{0}\right)=0\right\} .
$$

\subsection{A complete description of $x$-flat outputs for the nonholonomic $n$-trailer system}

Consider the kinematic model of a unicycle-like mobile robot towing $n$ trailers such that the towing hook of each trailer is located at the center of its unique axle (with the assumption that the distances between any two consecutive trailers are equal), shown in Figure 2. The $n$-trailer system is subject to nonholonomic constraints: it is assumed that the wheels of each individual trailer are aligned with the body and are not allowed to slip [16]. This model and its control properties have attracted a lot of attention (see the books $[17,18]$; and the papers $[6,14,24,26,30])$. We use here the following description introduced in [26].

Consider the $n$-trailer system $\Sigma_{\mathrm{tr}}^{\mathrm{n}}$ defined on $\mathbb{R}^{2} \times\left(S^{1}\right)^{n+1}$, for $n \geq 0$,

$$
\Sigma_{\mathrm{tr}}^{\mathrm{n}}: \dot{q}=f_{1}(q) u_{1}+f_{2}(q) u_{2}, \quad q \in \mathbb{R}^{2} \times\left(S^{1}\right)^{n+1},
$$

where the vector fields $f_{1}$ and $f_{2}$ are given by

$$
\begin{aligned}
& f_{1}=\pi_{0} \cos \theta_{0} \frac{\partial}{\partial x}+\pi_{0} \sin \theta_{0} \frac{\partial}{\partial y}+\sum_{i=0}^{n-1} \pi_{i+1} \sin \left(\theta_{i+1}-\theta_{i}\right) \frac{\partial}{\partial \theta_{i}} \\
& f_{2}=\frac{\partial}{\partial \theta_{n}}
\end{aligned}
$$

with $\pi_{i}=\prod_{j=i+1}^{n} \cos \left(\theta_{j}-\theta_{j-1}\right)$ and $\pi_{n}=1$. The configuration of this system is described by $q=\left(x, y, \theta_{0}, \ldots, \theta_{n}\right)$ $\in \mathbb{R}^{2} \times\left(S^{1}\right)^{n+1}$, where $(x, y)$ denotes the position of the last trailer while $\theta_{0}, \ldots, \theta_{n}$ represent the angles between each trailer's axle and the $x$-axis.

According to Theorem 1.2, the $n$-trailer system is locally $x$-flat at any $\left(q_{0}, u_{0}\right)$ such that $\operatorname{dim} \mathcal{D}^{(i)}\left(q_{0}\right)=$ $\operatorname{dim} \mathcal{D}_{i}\left(q_{0}\right), 0 \leq i \leq n$ (equivalently $\mathcal{D}$ is equivalent to the Goursat normal form around $\left.q_{0}\right)$ and $u_{0} \notin U_{\text {sing }}\left(q_{0}\right)$. The former condition yields $\cos \left(\theta_{i, 0}-\theta_{i-1,0}\right) \neq 0, i \geq 2$, and the latter means that $u_{0}=\left(u_{10}, u_{20}\right)$ satisfies 
$u_{10} \neq 0$. In other words, the $n$-trailer is $x$-flat along a trajectory $q(t)$ besides those time instances $t_{0}$ at which the angle between two consecutive trailers becomes $\pm \frac{\pi}{2}$ (except for the angle between the last trailer and one before the last which can be any) or those instances at which the velocity $\dot{q}\left(t_{0}\right)$ becomes parallel to $\frac{\partial}{\partial \theta_{n}}$, that is, the whole $n$-trailer movement stops.

Let $\mathcal{D}$ be the distribution associated to the $n$-trailer system $\Sigma_{\mathrm{tr}}^{n}$, that is $\mathcal{D}=\operatorname{span}\left\{f_{1}, f_{2}\right\}$. It is easy to check that $\operatorname{rank} \mathcal{D}^{(i)}=i+2$, for $0 \leq i \leq n+1$, and that, see e.g., Lemma 4.10 in [26], the characteristic distribution $\mathcal{C}_{i}$ of $\mathcal{D}^{(i)}$, for $1 \leq i \leq n$, is given by

$$
\mathcal{C}_{i}=\operatorname{span}\left\{c_{1}, \ldots, c_{i}\right\}=\operatorname{span}\left\{\frac{\partial}{\partial \theta_{n}}, \ldots, \frac{\partial}{\partial \theta_{n-i+1}}\right\} .
$$

Taking $g=f_{1}$ we have clearly $\mathcal{D}^{(i)}=\operatorname{span}\left\{c_{1}, \ldots, c_{i+1}, g\right\}$, for $1 \leq i \leq n-1$, around any point $q_{0}$ such that $\cos \left(\theta_{i, 0}-\theta_{i-1,0}\right) \neq 0$. According to the analysis performed in Section 2.2, and summarized in Theorem 2.10, as a first flat output around a given point $q_{0} \in \mathbb{R}^{2} \times\left(S^{1}\right)^{n+1}$, we can take any function $\varphi_{1}$ satisfying $L_{c_{i}} \varphi_{1}=\frac{\partial \varphi_{1}}{\partial \theta_{i}} \equiv 0$, for $1 \leq i \leq n$, and $L_{g} \varphi_{1}\left(q_{0}\right) \neq 0$, that is any function $\varphi_{1}=\varphi_{1}\left(x, y, \theta_{0}\right)$ such that $L_{g} \varphi_{1}\left(q_{0}\right) \neq 0$. Let us choose one such $\varphi_{1}$ and then $\varphi_{2}$ has to satisfy

$$
\begin{aligned}
& L_{c_{i} \varphi_{2}}=0, \quad 1 \leq i \leq n \\
& L_{v_{n} \varphi_{2}}=0
\end{aligned}
$$

where $v_{n}=\left(L_{g} \varphi_{1}\right)\left[c_{n}, g\right]-\left(L_{\left[c_{n}, g\right]} \varphi_{1}\right) g$ (notice that the dimension of the state space is $\left.n+3\right)$. The conditions $L_{c_{i}} \varphi_{2}=0,1 \leq i \leq n$, imply that $\varphi_{2}=\varphi_{2}\left(x, y, \theta_{0}\right)$ and now we consider the equation $L_{v_{n}} \varphi_{2}=0$. We have

$$
g=\eta \tilde{g}+\sum_{i=1}^{n-1} \pi_{i+1} \sin \left(\theta_{i+1}-\theta_{i}\right) c_{n-i+1},
$$

where $\eta=\prod_{i=1}^{n-1} \cos \left(\theta_{i+1}-\theta_{i}\right)$ and $\tilde{g}=\cos \left(\theta_{1}-\theta_{0}\right) \cos \theta_{0} \frac{\partial}{\partial x}+\cos \left(\theta_{1}-\theta_{0}\right) \sin \theta_{0} \frac{\partial}{\partial y}+\sin \left(\theta_{1}-\theta_{0}\right) \frac{\partial}{\partial \theta_{0}}$. Recall that around $q_{0}$ under consideration $\theta_{i+1,0}-\theta_{i, 0} \neq \pm \frac{\pi}{2}$. So $\eta \neq 0$ and by a direct calculation we check that $L_{v_{n}} \varphi_{2}=0$ if and only if $L_{\tilde{v}_{n}} \varphi_{2}=0$, where

$$
\begin{aligned}
\tilde{v}_{n} & =\left(L_{\tilde{g}} \varphi_{1}\right)\left[c_{n}, \tilde{g}\right]-\left(L_{\left[c_{n}, \tilde{g}\right]} \varphi_{1}\right) \tilde{g} \\
& =-\frac{\partial \varphi_{1}}{\partial \theta_{0}} \cos \theta_{0} \frac{\partial}{\partial x}-\frac{\partial \varphi_{1}}{\partial \theta_{0}} \sin \theta_{0} \frac{\partial}{\partial y}+\left(\frac{\partial \varphi_{1}}{\partial x} \cos \theta_{0}+\frac{\partial \varphi_{1}}{\partial y} \sin \theta_{0}\right) \frac{\partial}{\partial \theta_{0}}
\end{aligned}
$$

Given any $\varphi_{1}=\varphi_{1}\left(x, y, \theta_{0}\right)$ such that $L_{g} \varphi_{1}\left(q_{0}\right) \neq 0$, the space of solutions of $L_{\tilde{v}_{n}} \varphi_{2}=L_{c_{i}} \varphi_{2}=0$ is that of functions of two variables. Moreover any two solutions $\varphi_{2}$ and $\tilde{\varphi}_{2}$ give equivalent $x$-flat outputs $\operatorname{span}\left\{\mathrm{d} \varphi_{1}, \mathrm{~d} \varphi_{2}\right\}=\operatorname{span}\left\{\mathrm{d} \varphi_{1}, \mathrm{~d} \tilde{\varphi}_{2}\right\}=\mathcal{L}^{\perp}$, where $\mathcal{L}=\operatorname{span}\left\{c_{1}, \ldots, c_{n}, v\right\}=\operatorname{span}\left\{c_{1}, \ldots, c_{n}, \tilde{v}\right\}$ (as we have already discussed in Sect. 2.3). This equivalence is very easy to prove and so we omit it here. Therefore $\varphi_{2}$ can be taken as any function $\varphi_{2}=\varphi_{2}\left(x, y, \theta_{0}\right)$ satisfying $L_{\tilde{v}_{n}} \varphi_{2}=0$ and $\left(\mathrm{d} \varphi_{1} \wedge \mathrm{d} \varphi_{2}\right)\left(x_{0}\right) \neq 0$. The space of solutions is thus defined on $\{q: \eta(q) \neq 0\}$, i.e., on $\left\{q: \theta_{i+1}-\theta_{i} \neq \pm \frac{\pi}{2}, 1 \leq i \leq n-1\right\}$.

Take $\varphi_{1}=x$, then $\tilde{v}_{n}=\cos \theta_{0} \frac{\partial}{\partial \theta_{0}}$ and $L_{c_{i}} \varphi_{2}=L_{\tilde{v}_{n}} \varphi_{2}=0$, for $1 \leq i \leq n$, imply that $\varphi_{2}$ is any function of the form $\varphi_{2}(x, y)$ satisfying $\frac{\partial \varphi_{2}}{\partial y}\left(q_{0}\right) \neq 0$ (because of $\left.\left(\mathrm{d} \varphi_{1} \wedge \mathrm{d} \varphi_{2}\right)\left(q_{0}\right) \neq 0\right)$. All such functions satisfy $\operatorname{span}\left\{\mathrm{d} x, \mathrm{~d} \varphi_{2}\right\}=\operatorname{span}\left\{\mathrm{d} x, \mathrm{~d} \tilde{\varphi}_{2}\right\}$ and we can take, for instance, $\varphi_{2}=y$.

To see another choice, take $\varphi_{1}=\theta_{0}$, then $\tilde{v}_{n}=-\cos \theta_{0} \frac{\partial}{\partial x}-\sin \theta_{0} \frac{\partial}{\partial y}$ and the general solution of $L_{c_{i}} \varphi_{2}=$ $L_{\tilde{v}_{n}} \varphi_{2}=0$ is $\varphi_{2}=\varphi_{2}\left(\theta_{0}, x \sin \theta_{0}-y \cos \theta_{0}\right)$, and we can take, for instance $\varphi_{2}=x \sin \theta_{0}-y \cos \theta_{0}$. 
It is interesting to notice that the family of all $x$-flat outputs of the $n$-trailer system coincides with the family of all $x$-flat outputs of the nonholonomic car, more precisely of the car defined as the tail consisting of the last and one-before-the-last trailers (those indexed, respectively, by $i=0$ and $i=1$ ) controlled by towing (forward and backward) and rotating the one-before-the-last trailer. Indeed, the families of $x$-flat outputs coincide because they are given by any $\varphi_{1}=\varphi_{1}\left(x, y, \theta_{0}\right)$ and any $\varphi_{2}\left(x, y, \theta_{0}\right)$ satisfying $L_{\tilde{v}_{n}} \varphi_{2}=0$ (for the $n$-trailer) and $L_{v_{1}} \varphi_{2}=0$ (for the car) but, clearly, these two equations are equivalent (compare Sect. 3.1).

\section{Proof of main theorems}

\subsection{Useful results}

In this section we give a series of results that we will use in the subsequent sections when proving our theorems. We start with a weaker version of Proposition 1.3 proving that the statement of the latter holds on open and dense subset.

Lemma 4.1. Consider a driftless 2-input smooth control system $\Sigma$ defined on a manifold $M$ of dimension $n+2$. Let $\varphi_{1}, \varphi_{2}$ be two functions defined on $M$. If $\left(\varphi_{1}, \varphi_{2}\right)$ is an $x$-flat output of $\Sigma$ at $\left(x_{0}, u_{0}\right)$, then there exists an open neighborhood $\mathcal{M}$ of $x_{0}$ and an open and dense subset $\tilde{\mathcal{M}}$ of $\mathcal{M}$ such that $\varphi_{1}, \varphi_{2}$ are defined in $\mathcal{M}$ and around any point $q \in \tilde{\mathcal{M}}$ there exist coordinates $\left(z_{1}, \ldots, z_{n+2}\right)$, centered at $q$, in which $\Sigma$ is locally feedback equivalent to the chained form $\Sigma_{\text {chain, }}$ given by (1.3), such that $z_{1}=\varphi_{1}$ and $z_{2}=\varphi_{2}+k_{0} \varphi_{1}$, where $k_{0} \in \mathbb{R}$ is a constant (after permuting $\varphi_{1}$ and $\varphi_{2}$, if necessary).

Proof. Let $\left(\varphi_{1}, \varphi_{2}\right)$ be an $x$-flat output of $\Sigma$ at $\left(x_{0}, u_{0}\right)$. There exists an open neighborhood $\mathcal{M}$ of $x_{0}$ such that $\left(\varphi_{1}, \varphi_{2}\right)$ is an $x$-flat output at $(x, u)$ for any $x \in \mathcal{M}$ and $u=u(x)$. Recall that we can assume that $\varphi_{1}\left(x_{0}\right)=\varphi_{2}\left(x_{0}\right)=0$ (by replacing $\varphi_{i}$ by $\varphi_{i}-\varphi_{i}\left(x_{0}\right)$, if necessary). It is known (see, e.g., $\left.[7,13,28]\right)$ that the differentials of flat output are independent at $x_{0}$ and thus we put $x_{1}=\varphi_{1}, x_{2}=\varphi_{2}$, and complete them to a coordinate system $\xi=\left(x_{1}, x_{2}, \ldots, x_{n+2}\right)$. Consider the $(2 \times 2)$-matrix $\mathrm{D}=\left(\mathrm{D}_{i j}\right)$ given by $\mathrm{D}_{i j}=L_{g_{j}} \varphi_{i}$, $1 \leq i, j \leq 2$. It is immediate to see that $\operatorname{rank} \mathrm{D}(q) \leq 1$, for any $q \in \mathcal{M}$. Indeed, if the rank were two then by a suitable invertible feedback $u=\beta(x) v$ we would get

$$
\begin{array}{ll}
\varphi_{1}=x_{1}, & \dot{x}_{1}=v_{1} \\
\varphi_{2}=x_{2}, & \dot{x}_{2}=v_{2}
\end{array}
$$

which contradicts the flatness assumption because $\varphi_{i}^{(j)}=v_{i}^{(j-1)}$, for $1 \leq i \leq 2$, and any $j \geq 1$, and thus the coordinates $x_{3}, \ldots, x_{n+2}$ could not be represented as functions of $\varphi_{i}^{(j)}, j \geq 0$. Therefore on an open and dense subset $\mathcal{M}^{\prime}$ of $\mathcal{M}, \operatorname{rank} \mathrm{D}(q)=1$, for $q \in \mathcal{M}^{\prime}$. Fix $q \in \mathcal{M}^{\prime}$ and apply around $q$ a suitable invertible feedback $u=\beta(x) v$ in order to get (if necessary, we permute $\varphi_{1}$ and $\varphi_{2}$, and replace $\varphi_{i}$ by $\varphi_{i}-\varphi_{i}(q)$ )

$$
\begin{array}{ll}
x_{1}=\varphi_{1}, & \dot{x}_{1}=v_{1} \\
x_{2}=\varphi_{2}, & \dot{x}_{2}=\tilde{\psi}(x) v_{1}
\end{array}
$$

where $\tilde{\psi}(x)$ is a smooth function. Define $\tilde{\varphi}_{1}=\varphi_{1}, \tilde{\varphi}_{2}=\varphi_{2}-\tilde{\psi}(q) \varphi_{1}$ and clearly $\left(\tilde{\varphi}_{1}, \tilde{\varphi}_{2}\right)$ is also a flat output. We replace $x_{2}$ by $\tilde{\varphi}_{2}=x_{2}-\tilde{\psi}(q) x_{1}$ and then in the new coordinates, the system becomes

$$
\dot{x}=g_{1}(x) v_{1}+g_{2}(x) v_{2}
$$

where the flat outputs and their derivatives are

$$
\begin{array}{ll}
x_{1}=\tilde{\varphi}_{1}=\varphi_{1}, & \dot{x}_{1}=v_{1} \\
x_{2}=\tilde{\varphi}_{2}=\varphi_{2}+k_{0} \varphi_{1}, & \dot{x}_{2}=\psi(x) v_{1}=\left(\tilde{\psi}(x)+k_{0}\right) v_{1}
\end{array}
$$

where $k_{0}=-\tilde{\psi}(q)$ and $\psi(x)$ is a smooth function that satisfies $\psi(q)=0$. 
Consider the vector fields

$$
\begin{aligned}
& g_{1}=\frac{\partial}{\partial x_{1}}+\psi(x) \frac{\partial}{\partial x_{2}}+\sum_{i=3}^{n+2} g_{1 i}(x) \frac{\partial}{\partial x_{i}} \\
& g_{2}=\sum_{i=3}^{n+2} g_{2 i}(x) \frac{\partial}{\partial x_{i}} .
\end{aligned}
$$

We claim that for any $q$ in an open and dense subset $\mathcal{M}^{\prime \prime}$ of $\mathcal{M}^{\prime}$ there exists $\rho$ such that

$$
\begin{aligned}
& L_{g_{2}} L_{g_{1}}^{\mu} \psi \quad \equiv 0, \quad \text { for } 0 \leq \mu \leq \rho-2, \\
& L_{g_{2}} L_{g_{1}}^{\rho-1} \psi(q) \neq 0,
\end{aligned}
$$

and, moreover, that $\rho=n$ for any $q \in \mathcal{M}^{\prime \prime}$. In other words, $\rho=n$ is the relative degree of the single-input system $\dot{x}=f+v g$, where $f=g_{1}, g=g_{2}$ and $v=v_{2}$, equipped with the output $y=\psi$.

To prove our claim, first, observe that $\rho$ exists on an open and dense subset $\mathcal{M}^{\prime \prime}$ of $\mathcal{M}^{\prime}$. If not, on an open set in $\mathcal{M}^{\prime}$, we would have $L_{g_{2}} L_{g_{1}}^{\mu} \psi \equiv 0$ for any $\mu \geq 0$, which contradicts the flatness of $\Sigma$ since $v_{2}$ could not be expressed in terms of $\tilde{\varphi}_{i}^{(j)}, i=1,2, j \geq 0$. Thus $\rho$ exists and is locally constant on an open and dense subset $\mathcal{M}^{\prime \prime \prime}$ of $\mathcal{M}^{\prime \prime}$, with a priori different constant values on different connected components of $\mathcal{M}^{\prime \prime \prime}$. We claim that on each connected component the constant value of $\rho$ is $n$. On one hand, we have $\rho \leq n$ since $L_{g_{2}} \tilde{\varphi}_{1}=L_{g_{2}} \tilde{\varphi}_{2}=0$. On the other hand, if $\rho<n$, then we put $\tilde{v}_{2}=\left(L_{g_{2}} L_{g_{1}}^{\rho-1} \psi\right) v_{2}+\left(L_{g_{1}}^{\rho} \psi\right) v_{1}$ which is an invertible feedback, because of the definition of $\rho$. Now knowing $\tilde{\varphi}_{1}$ and $\tilde{\varphi}_{2}$, we can obtain $v_{1}=\dot{\tilde{\varphi}}_{1}$ and $\psi=\frac{\dot{\tilde{\varphi}}_{2}}{\dot{\tilde{\varphi}}_{1}}$, then $\rho-1$ functions $L_{g_{1}}^{\mu} \psi=\psi^{(\mu)}, 1 \leq \mu \leq \rho-1$, (by successive differentiations) and finally the control $\tilde{v}_{2}$. This gives two controls and $\rho+2<n+2$ functions, so one function among $x_{3}, \ldots, x_{n+2}$ is missing. This contradicts the flatness assumption. We thus have proved that on an open and dense subset $\mathcal{M}^{\prime \prime \prime}$ of $\mathcal{M}^{\prime}$, the relative degree $\rho$ is well defined and equals $n$.

Fix an arbitrary $q \in \mathcal{M}^{\prime \prime \prime}$, we can assume that $g_{1 i}(q)=0$, for $3 \leq i \leq n+2$ (if not, we replace $x_{i}$ by $x_{i}-k_{i} x_{1}$, for $3 \leq i \leq n+2$, where $k_{i}=g_{1 i}(q)$. We claim that the differentials of the functions $x_{1}, x_{2}, \psi, L_{g_{1}} \psi, \ldots, L_{g_{1}}^{n-1} \psi$ are independent at $q \in \mathcal{M}^{\prime \prime \prime}$. To this end, we will use the following result (see, e.g., Isidori [11]): if two vector fields $f$ and $g$ and a function $\phi$ satisfy

$$
L_{g} \phi=L_{g} L_{f} \phi=\cdots=L_{g} L_{f}^{k-2} \phi=0, \quad L_{g} L_{f}^{k-1} \phi=\lambda,
$$

where $\lambda$ is a function, then for any $1 \leq j \leq k-1$,

$$
L_{\mathrm{ad}_{f}^{j} g} \phi=L_{\mathrm{ad}_{f}^{j} g}\left(L_{f} \phi\right)=\cdots=L_{\mathrm{ad}_{f}^{j} g}\left(L_{f}^{k-j-2} \phi\right)=0, \quad L_{\mathrm{ad}_{f}^{j} g}\left(L_{f}^{k-j-1} \phi\right)=(-1)^{j} \lambda .
$$

We apply this result to $f=g_{1}, g=g_{2}, \phi=\psi$ and $k=n$ so $\lambda=L_{g_{2}} L_{g_{1}}^{n-1} \psi$ and, in particular, $\lambda(q) \neq 0$. Since, $L_{g_{2}} \tilde{\varphi}_{1}=0$ and $L_{g_{1}} \tilde{\varphi}_{1}=1$, it follows that

$$
L_{\mathrm{ad}_{g_{1}} g_{2}} \tilde{\varphi}_{1}=L_{\left[g_{1}, g_{2}\right]} \tilde{\varphi}_{1}=L_{g_{1}} L_{g_{2}} \tilde{\varphi}_{1}-L_{g_{2}} L_{g_{1}} \tilde{\varphi}_{1}=0
$$

and by induction we prove easily that $L_{\operatorname{ad}_{g_{1}}^{j} g_{2}} \tilde{\varphi}_{1}=0$. Moreover, $\psi=L_{g_{1}} \tilde{\varphi}_{2}$ implies that

$$
\begin{aligned}
L_{\mathrm{ad}_{g_{1}} g_{2}} \tilde{\varphi}_{2} & =\cdots=L_{\mathrm{ad}_{g_{1}}^{n-1} g_{2}} \tilde{\varphi}_{2}=0 \\
L_{\mathrm{ad}_{g_{1}}^{n} g_{2}} \tilde{\varphi}_{2}(q) & =(-1)^{n} L_{g_{2}} L_{g_{1}}^{n} \tilde{\varphi}_{2}(q)=(-1)^{n} L_{g_{2}} L_{g_{1}}^{n-1} \psi(q) \neq 0 .
\end{aligned}
$$


Evaluating the differential forms $\mathrm{d} \tilde{\varphi}_{i}$ and $\mathrm{d} L_{g_{1}}^{j} \psi, 0 \leq j \leq n-1$, on the vector fields $g_{2}, \operatorname{ad}_{g_{1}} g_{2}, \ldots, \operatorname{ad}_{g_{1}}^{n} g_{2}, g_{1}$ we get (notice that $L_{g_{1}} \tilde{\varphi}_{2}=\psi$ )

$$
\left(\begin{array}{c}
\mathrm{d} \tilde{\varphi}_{1} \\
\mathrm{~d} \tilde{\varphi}_{2} \\
\mathrm{~d} \psi \\
\mathrm{d} L_{g_{1}} \psi \\
\vdots \\
\mathrm{d} L_{g_{1}}^{n-1} \psi
\end{array}\right)\left(g_{2}, \operatorname{ad}_{g_{1}} g_{2}, \ldots, \operatorname{ad}_{g_{1}}^{n} g_{2}, g_{1}\right)=\left(\begin{array}{ccccc}
0 & 0 & \cdots & 0 & 1 \\
0 & 0 & \cdots & (-1)^{n} \lambda & \psi \\
\cdots & \cdots & \cdots & * & * \\
0 & -\lambda & \cdots & \cdots & \cdots \\
\lambda & * & \cdots & \cdots & *
\end{array}\right)
$$

The determinant of the above matrix is nonzero since $\lambda(q) \neq 0$, and therefore the functions $\tilde{\varphi}_{1}, \tilde{\varphi}_{2}, \psi, L_{g_{1}} \psi, \ldots$, $L_{g_{1}}^{n-1} \psi$ are independent. It follows that

$$
\begin{aligned}
z_{1} & =\tilde{\varphi}_{1} \\
z_{2} & =\tilde{\varphi}_{2} \\
z_{3} & =\psi \\
z_{4} & =L_{g_{1}} \psi \\
& \vdots \\
z_{n+2} & =L_{g_{1}}^{n-1} \psi
\end{aligned}
$$

is a valid local change of coordinates in a neighborhood of any $q \in \tilde{\mathcal{M}}=\mathcal{M}^{\prime \prime \prime}$, centered at $q$, in which the system after applying the feedback $\tilde{v}_{1}=v_{1}, \tilde{v}_{2}=\left(L_{g_{1}}^{n} \psi\right) v_{1}+\left(L_{g_{2}} L_{g_{1}}^{n-1} \psi\right) v_{2}$ (invertible since $\left.\left(L_{g_{2}} L_{g_{1}}^{n-1} \psi\right)(q) \neq 0\right)$ takes the chained form $\Sigma_{\text {chain }}$ while the top two variables are given by $z_{1}=\tilde{\varphi}_{1}=\varphi_{1}$ and $z_{2}=\tilde{\varphi}_{2}=\varphi_{2}+k_{0} \varphi_{1}$, with $k_{0}=-\tilde{\psi}(q)=-\frac{\dot{\varphi}_{2}}{\dot{\varphi}_{1}}(q) \in \mathbb{R}$.

Although the above lemma was proved at generic points only, it implies the following result in a whole neighborhood of the point $x_{0}$ under consideration. Recall that for any Goursat structure $\mathcal{D}$ we denote by $\mathcal{C}_{n-1}$ the characteristic distribution of $\mathcal{D}^{(n-1)}$ (see Lem. 2.1).

Corollary 4.2. Consider a Goursat structure $\mathcal{D}$ on $M$ of dimension $n+2$, that is, $\operatorname{rank} \mathcal{D}^{(i)}=i+2$, for $0 \leq$ $i \leq n$, hold everywhere on $M$. If the associated control system $\Sigma$ is $x$-flat at $\left(x_{0}, u_{0}\right) \in M \times \mathbb{R}^{2}, u_{0} \notin U_{\text {sing }}\left(x_{0}\right)$, then for any $x$-flat output $\left(\varphi_{1}, \varphi_{2}\right)$ at $\left(x_{0}, u_{0}\right)$, there exists an open neighborhood $\mathcal{M}$ of $x_{0}$ in which we have $L_{c} \varphi_{i}=0$, for $i=1,2$ and any $c \in \mathcal{C}_{n-1}$.

Proof. Let $\left(\varphi_{1}, \varphi_{2}\right)$ be an $x$-flat output of $\Sigma$ at $\left(x_{0}, u_{0}\right)$. By Lemma 4.1, there exists an open neighborhood $\mathcal{M}$ (where $\varphi_{1}, \varphi_{2}$ are defined) of $x_{0}$ and an open and dense subset $\tilde{\mathcal{M}}$ of $\mathcal{M}$ such that around any $q \in \tilde{\mathcal{M}}$, the system $\Sigma$ is feedback equivalent to the chained form $\Sigma_{\text {chain }}$, given by (1.3), with $z_{1}=\varphi_{1}$ and $z_{2}=\varphi_{2}+k_{0} \varphi_{1}$ (after permuting $\varphi_{1}$ and $\varphi_{2}$, if necessary), where $k_{0} \in \mathbb{R}$ is a constant. We have $\mathcal{C}_{n-1}=\operatorname{span}\left\{\frac{\partial}{\partial z_{4}}, \ldots, \frac{\partial}{\partial z_{n+2}}\right\}$ and hence $L_{c} \varphi_{i}=0$, for $i=1,2$ and any $c \in \mathcal{C}_{n-1}$ on $\tilde{\mathcal{M}}$ and hence on $\mathcal{M}$ (since $\tilde{\mathcal{M}}$ is dense and the functions $\varphi_{i}$ as well as the distribution $\mathcal{C}_{n-1}$ are well defined on the whole $\mathcal{M}$ ).

\subsection{Proof of Theorem $\mathbf{1 . 2}$}

We will show the implications (iii) $\Rightarrow$ (iv) $\Rightarrow$ (ii) $\Rightarrow$ (i) $\Rightarrow$ (iii).

(iii) $\Rightarrow$ (iv): It is a well known result (proved by Murray in [23]) that $\operatorname{dim} \mathcal{D}^{(i)}\left(x_{0}\right)=\operatorname{dim} \mathcal{D}_{i}\left(x_{0}\right)=i+2$, $0 \leq i \leq n$, are necessary and sufficient for local feedback equivalence to the chained form.

(iv) $\Rightarrow$ (ii): It is obvious for a system in the chained form $\Sigma_{\text {chain }}$, given by (1.3), that $\varphi_{1}=z_{1}$ and $\varphi_{2}=z_{2}$ yield flatness for $v_{1} \neq 0$ and the latter means that we can take in (ii) any $u_{0} \notin U_{\text {sing }}\left(x_{0}\right)$. To see that $v_{1}=0$ is 
not a singular control, if $n=1$, introduce the new coordinate $\tilde{z}_{2}=z_{2}-z_{1} z_{3}$ to transform the chain form into

$$
\begin{aligned}
& \dot{z}_{1}=v_{1} \\
& \dot{\tilde{z}}_{2}=-z_{1} v_{2} \\
& \dot{z}_{3}=v_{2}
\end{aligned}
$$

for which $\left(\tilde{\varphi}_{1}, \tilde{\varphi}_{2}\right)=\left(\tilde{z}_{2}, z_{3}\right)$ is an $x$-flat output at $\left(z_{0}, v_{0}\right)$ such that $v_{20} \neq 0$. It follows that for $n=1$, i.e., $\operatorname{dim} M=3$, the singular control is $U_{\text {sing }}=(0,0)$ only.

(ii) $\Rightarrow$ (i): Obvious.

(i) $\Rightarrow$ (iii): This is the only difficult implication. Its proof will be based on Corollary 4.2 and on the result that assures that any Goursat structure can be brought into the following polynomial normal form, called Kumpera-Ruiz normal form, as proved by Pasillas-Lépine and Respondek [26] (see also Cheaito and Mormul [4], Mormul [22] and Kumpera and Ruiz [15]):

Theorem 4.3 (Kumpera-Ruiz normal form). Assume that $n \geq 2$. Any Goursat structure defined on a manifold $M$ of dimension $n+2$ is locally equivalent, at any point $x_{0}$ in $M$, to a distribution spanned in a small neighborhood of zero by a pair of vector fields that have the following form:

$$
f_{1}=\sum_{i=0}^{m}\left(\prod_{j=0}^{i-1} x_{k_{j}}^{j}\right)\left(\sum_{j=1}^{k_{i}-1}\left(x_{j}^{i}+c_{j}^{i}\right) \frac{\partial}{\partial x_{j+1}^{i}}+\frac{\partial}{\partial x_{1}^{i+1}}\right), \quad f_{2}=\frac{\partial}{\partial x_{1}^{0}},
$$

where the coordinates $x_{j}^{i}$, for $0 \leq i \leq m+1$ and $1 \leq j \leq k_{i}$, are centered at $x_{0}$ (that means that $x\left(x_{0}\right)=0$ ); the integer $m$ is such that $0 \leq m \leq n-2$; and $k_{i}$, for $0 \leq i \leq m-1$, satisfy $k_{0} \geq 1, \ldots, k_{m-1} \geq 1, k_{m} \geq 3$, $k_{m+1}=1$ and $\sum_{i=0}^{m+1} k_{i}=n+2$; the constants $c_{j}^{i}$, for $1 \leq j \leq k_{i}-1$, are real constants.

Remark 4.4. In the above normal form, the integer $m$ gives the number of singularities of the Kumpera-Ruiz normal form. When $m=0$, the Kumpera-Ruiz normal form coincides with the Goursat normal form (since in this case all constants $c_{j}^{i}$ can be eliminated).

In order to prove (i) $\Rightarrow$ (iii), assume that $\Sigma$ is $x$-flat at $\left(x_{0}, \bar{u}_{0}^{l}\right)$, for a certain $l \geq 0$, and that there exists an integer $2 \leq i \leq n$ such that $\operatorname{dim} \mathcal{D}^{(i)}\left(x_{0}\right) \neq \operatorname{dim} \mathcal{D}_{i}\left(x_{0}\right)$. Since $\mathcal{D}$ is a Goursat structure and $\operatorname{dim} \mathcal{D}^{(i)}\left(x_{0}\right) \neq \operatorname{dim} \mathcal{D}_{i}\left(x_{0}\right)$, for certain $2 \leq i \leq n$, by Theorem 4.3 , there exists a new coordinate system $\left(x_{1}^{0}, \ldots, x_{k_{0}}^{0}, \ldots, x_{1}^{m}, \ldots, x_{k_{m}}^{m}, x_{1}^{m+1}\right)$ in which $\mathcal{D}$ takes, in a small neighborhood of zero, the Kumpera-Ruiz normal form with $m \geq 1$ and $\sum_{i=0}^{m} k_{i}=n+1$, i.e., $\mathcal{D}=\operatorname{span}\left\{f_{1}, f_{2}\right\}$ where $f_{1}$ and $f_{2}$ are given by (4.14). A direct calculation shows that $\mathcal{D}^{(j)}=\operatorname{span}\left\{\frac{\partial}{\partial x_{1}^{0}}, \ldots, \frac{\partial}{\partial x_{j+1}^{0}}, f_{1}\right\}, \quad 1 \leq j \leq k_{0}-1$, and $\mathcal{C}_{j}=\operatorname{span}\left\{\frac{\partial}{\partial x_{1}^{0}}, \ldots, \frac{\partial}{\partial x_{j}^{0}}\right\}$, $1 \leq j \leq k_{0}-1$. Observing that

$$
\left[\frac{\partial}{\partial x_{k_{0}}^{0}}, f_{1}\right]=\sum_{i=1}^{m}\left(\prod_{j=1}^{i-1} x_{k_{j}}^{j}\right)\left(\sum_{j=1}^{k_{i}-1}\left(x_{j}^{i}+c_{j}^{i}\right) \frac{\partial}{\partial x_{j+1}^{i}}+\frac{\partial}{\partial x_{1}^{i+1}}\right)
$$

and

we then get

$$
\frac{\partial}{\partial x_{1}^{1}}=f_{1}-x_{k_{0}}^{0}\left[\frac{\partial}{\partial x_{k_{0}}^{0}}, f_{1}\right]-\sum_{j=1}^{k_{0}-1}\left(x_{j}^{0}+c_{j}^{0}\right) \frac{\partial}{\partial x_{i+1}^{0}}
$$

$$
\begin{aligned}
\mathcal{D}^{\left(k_{0}\right)} & =\mathcal{D}^{\left(k_{0}-1\right)}+\left[\mathcal{D}^{\left(k_{0}-1\right)}, \mathcal{D}^{\left(k_{0}-1\right)}\right] \\
& =\operatorname{span}\left\{\frac{\partial}{\partial x_{1}^{0}}, \ldots, \frac{\partial}{\partial x_{k_{0}}^{0}}, \frac{\partial}{\partial x_{1}^{1}}, \sum_{i=1}^{m}\left(\prod_{j=1}^{i-1} x_{k_{j}}^{j}\right)\left(\sum_{j=1}^{k_{i}-1}\left(x_{j}^{i}+c_{j}^{i}\right) \frac{\partial}{\partial x_{j+1}^{i}}+\frac{\partial}{\partial x_{1}^{i+1}}\right)\right\},
\end{aligned}
$$


and $\mathcal{C}_{k_{0}}=\operatorname{span}\left\{\frac{\partial}{\partial x_{1}^{0}}, \ldots, \frac{\partial}{\partial x_{k_{0}}^{0}}\right\}$. In the same way, we obtain that $\mathcal{C}_{k_{i}+j}=\mathcal{C}_{k_{i}} \oplus \operatorname{span}\left\{\frac{\partial}{\partial x_{1}^{i+1}}, \ldots, \frac{\partial}{\partial x_{j}^{i+1}}\right\}$, for $0 \leq i \leq m, 1 \leq j \leq k_{i}$ and $k_{m}+j \leq n-1$. Therefore, finally, the characteristic distribution $\mathcal{C}_{n-1}$ of $\mathcal{D}^{(n-1)}$ is given by

$$
\mathcal{C}_{n-1}=\operatorname{span}\left\{\frac{\partial}{\partial x_{1}^{0}}, \ldots, \frac{\partial}{\partial x_{k_{0}}^{0}}, \ldots, \frac{\partial}{\partial x_{1}^{m}}, \ldots, \frac{\partial}{\partial x_{k_{m}-2}^{m}}\right\}=\left(\operatorname{span}\left\{\mathrm{d} x_{k_{m}-1}^{m}, \mathrm{~d} x_{k_{m}}^{m}, \mathrm{~d} x_{1}^{m+1}\right\}\right)^{\perp} .
$$

For simplicity, we denote the coordinates $x_{k_{m}-1}^{m}, x_{k_{m}}^{m}, x_{1}^{m+1}$ by $y_{1}, y_{2}, y_{3}$, respectively, and denote the remaining $n-1$ coordinates $x_{j}^{i}$ by $x_{1}, \ldots, x_{n-1}$, that is,

$$
(x, y)=\left(x_{1}, \ldots, x_{n-1}, y_{1}, y_{2}, y_{3}\right)=\left(x_{1}^{0}, \ldots, x_{k_{0}}^{0}, x_{1}^{1} \ldots, x_{1}^{m}, \ldots, x_{k_{m}-1}^{m}, x_{k_{m}}^{m}, x_{1}^{m+1}\right) .
$$

In $(x, y)$-coordinates, the control system $\Sigma$ associated to the Kumpera-Ruiz normal form (4.14), reads as

$$
\Sigma_{\mathrm{KR}}:\left\{\begin{aligned}
\dot{x_{1}} & =u_{1} \\
\dot{x_{2}} & =\gamma_{2}(x, y) u_{2} \\
& \vdots \\
\dot{x}_{n-1} & =\gamma_{n-1}(x, y) u_{2} \\
\dot{y_{1}} & =x_{r} \beta_{1}(x, y) u_{2} \\
\dot{y_{2}} & =x_{r} \beta_{2}(x, y) u_{2} \\
\dot{y_{3}} & =x_{r} \beta_{3}(x, y) u_{2}
\end{aligned}\right.
$$

where $\beta_{1}, \beta_{2}, \beta_{3}$ and $\gamma_{j}$, for $2 \leq j \leq n-1$, are smooth functions defined in a neighborhood of $0 \in \mathbb{R}^{n+2}$, $1 \leq r \leq n-1$ is an integer, and the characteristic distribution $\mathcal{C}_{n-1}$ is given by $\mathcal{C}_{n-1}=\operatorname{span}\left\{\frac{\partial}{\partial x_{1}}, \ldots, \frac{\partial}{\partial x_{n-1}}\right\}=$ $\left(\operatorname{span}\left\{\mathrm{d} y_{1}, \mathrm{~d} y_{2}, \mathrm{~d} y_{3}\right\}\right)^{\perp}$. By our assumption, $\Sigma_{\mathrm{KR}}$ is $x$-flat at $\left(0, \bar{u}_{0}^{l}\right) \in \mathbb{R}^{n+2} \times \mathbb{R}^{2(l+1)}$ and let $\left(\varphi_{1}, \varphi_{2}\right)$ be an $x$-flat output defined in a neighborhood $\mathcal{O}$ of $0 \in \mathbb{R}^{n+2}$. Being a Goursat structure, $\Sigma_{\mathrm{KR}} \operatorname{satisfies} \operatorname{dim} \mathcal{D}^{(i)}(z)=$ $\operatorname{dim} \mathcal{D}_{i}(z), 0 \leq i \leq n$, for any $z=(x, y)$ in an open and dense subset $\mathcal{O}^{\prime}$ of $\mathcal{O}$ and by Corollary 4.2 and the form of $\mathcal{C}_{n-1}$, we conclude that $\frac{\partial \varphi_{1}}{\partial x_{i}}=\frac{\partial \varphi_{2}}{\partial x_{i}}=0,1 \leq i \leq n-1$, holds in $\mathcal{O}^{\prime}$ and, since $\mathcal{O}^{\prime}$ is dense in $\mathcal{O}$, also in $\mathcal{O}$. It follows that $\varphi_{i}=\varphi_{i}\left(y_{1}, y_{2}, y_{3}\right)$ for $i=1,2$. Moreover, the fact that $\left(\varphi_{1}, \varphi_{2}\right)$ is an $x$-flat output at $\left(0, \bar{u}_{0}^{l}\right)$ implies that $\varphi_{1}, \varphi_{2}, \dot{\varphi}_{1}, \dot{\varphi}_{2}$ must be independent at $\left(0, u_{0}\right) \in \mathbb{R}^{n+2} \times \mathbb{R}^{2}$, where $u_{0}=\left(u_{10}, u_{20}\right)$, (see, e.g., [7,28]). Calculating the derivatives $\dot{\varphi}_{i}$, for $i=1,2$, we get

$$
\dot{\varphi}_{i}=\sum_{j=1}^{3} x_{r} \beta_{j} u_{2} \frac{\partial \varphi_{i}}{\partial y_{j}}=F_{i}\left(x, y_{1}, y_{2}, y_{3}, u_{2}\right), \quad i=1,2
$$

Then

$$
\mathrm{d} \dot{\varphi}_{i}=\mathrm{d} F_{i}=\sum_{j=1}^{n-1} \frac{\partial F_{i}}{\partial x_{j}} \cdot \mathrm{d} x_{j}+\sum_{j=1}^{3} \frac{\partial F_{i}}{\partial y_{j}} \cdot \mathrm{d} y_{j}+\frac{\partial F_{i}}{\partial u_{2}} \cdot \mathrm{d} u_{2}, \quad i=1,2
$$

and at $\left(0, u_{0}\right)$,

$$
\mathrm{d} \dot{\varphi}_{i}\left(0, u_{0}\right)=\mathrm{d} F_{i}\left(0, u_{0}\right)=\frac{\partial F_{i}}{\partial x_{r}}\left(0, u_{0}\right) \cdot \mathrm{d} x_{r}, \quad i=1,2,
$$

which implies that $\left(\mathrm{d} \varphi_{1} \wedge \mathrm{d} \varphi_{2} \wedge \mathrm{d} \dot{\varphi}_{1} \wedge \mathrm{d} \dot{\varphi}_{2}\right)\left(0, u_{10}, u_{20}\right)=0$, independently of the values of $u_{10}$ and $u_{20}$, which gives a contradiction. Therefore if a system associated to a Goursat structure is $x$-flat at $\left(x, \bar{u}_{0}^{l}\right)$, for some $\bar{u}_{0}^{l}$, then we have $\operatorname{dim} \mathcal{D}^{(i)}\left(x_{0}\right)=\operatorname{dim} \mathcal{D}_{i}\left(x_{0}\right), 0 \leq i \leq n$. 


\subsection{Proof of Theorem $\mathbf{2 . 2}$}

Proof. Sufficiency: Take any 2-input system whose associated distribution satisfies $\operatorname{dim} \mathcal{D}^{(i)}(x)=\operatorname{dim} \mathcal{D}_{i}(x)=$ $i+2$ everywhere in a neighborhood of $x_{0}$ and choose two functions fulfilling (i) - (iii). We can bring $\Sigma$ to the

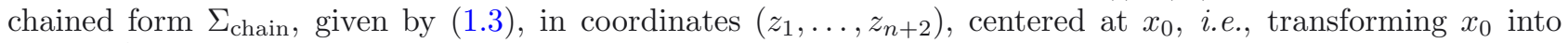
$0 \in \mathbb{R}^{n+2}$, and its associated distribution is given by $\mathcal{D}=\operatorname{span}\left\{g_{1}, g_{2}\right\}$, where

$$
\begin{aligned}
& g_{1}=\frac{\partial}{\partial z_{1}}+z_{3} \frac{\partial}{\partial z_{2}}+\cdots+z_{n+2} \frac{\partial}{\partial z_{n+1}} \\
& g_{2}=\frac{\partial}{\partial z_{n+2}} .
\end{aligned}
$$

The characteristic distribution $\mathcal{C}_{n-1}$ is given by $\mathcal{C}_{n-1}=\operatorname{span}\left\{\frac{\partial}{\partial z_{4}}, \ldots, \frac{\partial}{\partial z_{n+2}}\right\}$, and the condition $L_{c} \varphi_{i}=0$, for any $c \in \mathcal{C}_{n-1}$, of item (ii) implies that $\varphi_{i}=\varphi_{i}\left(z_{1}, z_{2}, z_{3}\right)$, for $i=1,2$. We assume $\varphi_{1}(0)=\varphi_{2}(0)=0$ (if not, we replace $\varphi_{i}$ by $\varphi_{i}-\varphi_{i}(0)$, for $i=1,2$, see Sect. 1 ). Item (iii) implies that there exists $\varphi_{i}$ ( $\operatorname{say} \varphi_{1}$, if not, we permute) such that $L_{g_{1}} \varphi_{1}(0)=\frac{\partial \varphi_{1}}{\partial z_{1}}(0) \neq 0$. Due to (i) we can complete $\varphi_{1}$ and $\varphi_{2}$ by a function $\varphi_{3}\left(z_{1}, z_{2}, z_{3}\right)$ such that $\left(\mathrm{d} \varphi_{1} \wedge \mathrm{d} \varphi_{2} \wedge \mathrm{d} \varphi_{3}\right)(0) \neq 0$ and we have $\dot{\varphi}_{i}=\psi_{i}\left(z_{1}, z_{2}, z_{3}, z_{4}\right) u_{1}, 1 \leq i \leq 3$, where $\psi_{i}=L_{g_{1}} \varphi_{i}$. Since $\psi_{1}(0)=L_{g_{1}} \varphi_{1}(0) \neq 0$, we introduce new coordinates $\tilde{z}_{1}=\varphi_{1}, \tilde{z}_{2}=\varphi_{2}-\frac{\psi_{2}}{\psi_{1}}(0) \varphi_{1}, \tilde{z}_{3}=\varphi_{3}-\frac{\psi_{3}}{\psi_{1}}(0) \varphi_{1}$, followed by $\tilde{z}_{i}=z_{i}, 4 \leq i \leq n$ and apply the invertible feedback $\tilde{u}_{1}=\psi_{1} u_{1}$ to get

$$
\Sigma:\left\{\begin{array}{l}
\dot{\tilde{z}}_{1}=\tilde{u}_{1} \\
\tilde{\tilde{z}}_{2}=\tilde{\psi}_{2} \tilde{u}_{1} \\
\dot{\tilde{z}}_{3}=\tilde{\psi}_{3} \tilde{u}_{1} \\
\dot{\tilde{z}}_{i}=\tilde{z}_{i+1} \frac{\tilde{u}_{1}}{\psi_{1}}, \quad \text { for } \quad 4 \leq i \leq n+1 \\
\dot{\tilde{z}}_{n+2}=u_{2},
\end{array}\right.
$$

where $\tilde{\psi}_{i}=\frac{\psi_{i}}{\psi_{1}}-\frac{\psi_{i}}{\psi_{1}}(0)$ satisfy $\tilde{\psi}_{i}(0)=0$, for $2 \leq i \leq 3$. Notice that the characteristic distribution $\mathcal{C}_{n-1}=$ $\operatorname{span}\left\{\frac{\partial}{\partial \tilde{z}_{4}}, \ldots, \frac{\partial}{\partial \tilde{z}_{n+2}}\right\}$ (since $\tilde{z}_{i}=\psi_{i}\left(z_{1}, z_{2}, z_{3}\right)$, for $\left.1 \leq i \leq 3\right)$. It thus follows from $L_{c}\left(\frac{L_{g_{1}} \varphi_{2}}{L_{g_{1}} \varphi_{1}}\right)=0$, $c \in \mathcal{C}_{n-1}$, that $\tilde{\psi}_{2}=\frac{\psi_{2}\left(z_{1}, z_{2}, z_{3}, z_{4}\right)}{\psi_{1}\left(z_{1}, z_{2}, z_{3}, z_{4}\right)}-\frac{\psi_{2}}{\psi_{1}}(0)$ is actually a function of $\tilde{z}_{1}, \tilde{z}_{2}, \tilde{z}_{3}$ only. Moreover, the condition $\operatorname{rank} \mathcal{D}^{(n)}=\operatorname{rank} \mathcal{D}_{n}=n+2$ implies that $\frac{\partial \tilde{\psi}_{3}}{\partial \tilde{z}_{4}}(0) \neq 0$ and a direct calculation shows that $L_{\tilde{g}_{2}} L_{\tilde{g}_{1}}^{\mu} \tilde{\psi}_{2} \equiv 0$ for $0 \leq \mu \leq n-2$ and $L_{\tilde{g}_{2}} L_{\tilde{g}_{1}}^{n-1} \tilde{\psi}_{2}(0) \neq 0$, where

$$
\begin{aligned}
& \tilde{g}_{1}=\frac{\partial}{\partial \tilde{z}_{1}}+\tilde{\psi}_{2} \frac{\partial}{\partial \tilde{z}_{2}}+\tilde{\psi}_{3} \frac{\partial}{\partial \tilde{z}_{3}}+\frac{\tilde{z}_{5}}{\psi_{1}} \frac{\partial}{\partial \tilde{z}_{4}}+\cdots+\frac{\tilde{z}_{n+2}}{\psi_{1}} \frac{\partial}{\partial \tilde{z}_{n+1}} \\
& \tilde{g}_{2}=\frac{\partial}{\partial \tilde{z}_{n+2}} .
\end{aligned}
$$

Therefore the function $\tilde{\psi}_{2}$ satisfies the conditions of $\psi$ from the proof of Lemma 4.1, and following that proof we can thus bring the system into the chained form (1.3), with $\left(\tilde{z}_{1}, \tilde{z}_{2}\right)=\left(\varphi_{1}, \varphi_{2}+k_{0} \varphi_{1}\right)$, where $k_{0}=-\frac{\psi_{2}}{\psi_{1}}(0) \in \mathbb{R}$, which proves that $\left(\varphi_{1}, \varphi_{2}+k_{0} \varphi_{1}\right)$ is indeed an $x$-flat output at $\left(x_{0}, u_{0}\right), u_{0} \notin U_{\text {sing }}\left(x_{0}\right)$ and so is $\left(\varphi_{1}, \varphi_{2}\right)$.

Necessity: Assume that $\Sigma$ is $x$-flat at $\left(x_{0}, u_{0}\right), u_{0} \notin U_{\text {sing }}\left(x_{0}\right)$, and let $\left(\varphi_{1}, \varphi_{2}\right)$ be an $x$-flat output defined in a neighborhood $\mathcal{M}$ of $x_{0}$. It is well known (see [7,13,28]) that $\mathrm{d} \varphi_{1}\left(x_{0}\right) \wedge \mathrm{d} \varphi_{2}\left(x_{0}\right) \neq 0$. By Lemma 4.1, we

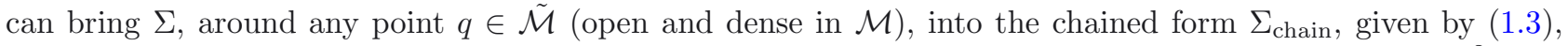
with $z_{1}=\varphi_{1}, z_{2}=\varphi_{2}+k_{0} \varphi_{1}$, where $k_{0} \in \mathbb{R}$ is a constant, and $q$ is transformed into $z_{0}=0 \in \mathbb{R}^{n+2}$. We have $\mathcal{C}_{n-1}=\operatorname{span}\left\{\frac{\partial}{\partial z_{4}}, \ldots, \frac{\partial}{\partial z_{n+2}}\right\}$. By a direct calculation we get that $L_{g} \varphi_{1}(0) \neq 0$ for any $g \in \mathcal{D}$ such that 
$g(0) \notin \mathcal{C}_{n-1}(0)$, and that $L_{c} \varphi_{1} \equiv L_{c} \varphi_{2} \equiv L_{c}\left(\frac{L_{g} \varphi_{2}}{L_{g} \varphi_{1}}\right) \equiv 0$, for any $c \in \mathcal{C}_{n-1}$ and $g$ as above, which gives the item (ii) on $\tilde{\mathcal{M}}$. Now observe that the flat outputs $\varphi_{1}, \varphi_{2}$ are well defined in $\mathcal{M}$ and so is the characteristic distribution $\mathcal{C}_{n-1}$ (since the distribution $\mathcal{D}$ associated to $\Sigma$ satisfies rank $\mathcal{D}^{(i)}=i+2$, for $0 \leq i \leq n$, everywhere in $\mathcal{M})$. It follows by continuity that $L_{c} \varphi_{1} \equiv L_{c} \varphi_{2} \equiv\left(L_{g} \varphi_{1}\right)\left(L_{c} L_{g} \varphi_{2}\right)-\left(L_{g} \varphi_{2}\right)\left(L_{c} L_{g} \varphi_{1}\right) \equiv 0$ holds also on $\mathcal{M}$ thus implying (ii) on $\mathcal{M}$.

It remains to prove (iii). Bring $\Sigma$, locally around $x_{0} \in \mathcal{M}$, into the chained form $\Sigma_{\text {chain }}$ given by (1.3), which is always possible by the assumption of theorem. Then item (ii), which we have just proved on $\mathcal{M}$, implies that $\varphi_{i}=\varphi_{i}\left(z_{1}, z_{2}, z_{3}\right)$, for $1 \leq i \leq 2$, and $g=\frac{\partial}{\partial z_{1}}+z_{3} \frac{\partial}{\partial z_{2}}+z_{4} \frac{\partial}{\partial z_{3}} \bmod \mathcal{C}_{n-1}$. If for $i=1$ or 2 , we have $L_{g} \varphi_{i}(0) \neq 0$, then (iii) holds. So assume that $L_{g} \varphi_{1}(0)=L_{g} \varphi_{2}(0)=0$ implying $\frac{\partial \varphi_{1}}{\partial z_{1}}(0)=\frac{\partial \varphi_{2}}{\partial z_{1}}(0)=0$. We have

$$
\begin{aligned}
& \dot{\varphi}_{1}=\left(\frac{\partial \varphi_{1}}{\partial z_{1}}+z_{3} \frac{\partial \varphi_{1}}{\partial z_{2}}+z_{4} \frac{\partial \varphi_{1}}{\partial z_{3}}\right) u_{1}=\left(a+b z_{4}\right) u_{1} \\
& \dot{\varphi}_{2}=\left(\frac{\partial \varphi_{2}}{\partial z_{1}}+z_{3} \frac{\partial \varphi_{2}}{\partial z_{2}}+z_{4} \frac{\partial \varphi_{2}}{\partial z_{3}}\right) u_{1}=\left(c+d z_{4}\right) u_{1}
\end{aligned}
$$

and thus $\dot{\varphi}_{1}\left(0, u_{0}\right)=\dot{\varphi}_{2}\left(0, u_{0}\right)=0$, where $u_{0}=\left(u_{10}, u_{20}\right)$ is the arbitrary nominal control (recall that the $z$ coordinates are centered at zero). We have $\frac{\partial \dot{\varphi}_{1}}{\partial u_{1}}(0)=\frac{\partial \dot{\varphi}_{2}}{\partial u_{1}}(0)=0$ and, since $\left(\mathrm{d} \varphi_{1} \wedge \mathrm{d} \varphi_{2} \wedge \mathrm{d} \dot{\varphi}_{1} \wedge \mathrm{d} \dot{\varphi}_{2}\right)\left(0, u_{0}\right) \neq 0$, it follows that either $\frac{\partial \dot{\varphi}_{1}}{\partial z_{4}}\left(0, u_{0}\right) \neq 0$ or $\frac{\partial \dot{\varphi}_{2}}{\partial z_{4}}\left(0, u_{0}\right) \neq 0$. Therefore $u_{10} \neq 0$ and we can assume that $b(0) \neq 0$ (if $d(0) \neq 0$, then we permute $\varphi_{1}$ and $\left.\varphi_{2}\right)$. The condition $\left(L_{g} \varphi_{1}\right)\left(L_{c} L_{g} \varphi_{2}\right)-\left(L_{g} \varphi_{2}\right)\left(L_{c} L_{g} \varphi_{1}\right) \equiv 0$, that we have already proved, gives $a d \equiv c b$ implying that the vectors $(a, b)$ and $(c, d)$ are collinear and, since $b(0) \neq 0$, there exists a smooth function $\eta=\eta\left(z_{1}, z_{2}, z_{3}\right)$ such that $(c, d)=\eta(a, b)$ implying that $\dot{\varphi}_{1}=\left(a+b z_{4}\right) u_{1}$ and $\dot{\varphi}_{2}=\eta\left(a+b z_{4}\right) u_{1}$. It follows (recall that $\left.\left(a+b z_{4}\right)(0)=0\right)$ that $\mathrm{d} \dot{\varphi}_{1}\left(0, u_{0}\right)=u_{10} \mathrm{~d}\left(a+b z_{4}\right)(0)$ and $\mathrm{d} \dot{\varphi}_{2}\left(0, u_{0}\right)=u_{10} \eta(0) \mathrm{d}\left(a+b z_{4}\right)(0)$ thus contradicting the linear independence of $\mathrm{d} \dot{\varphi}_{1}(0)$ and $\mathrm{d} \dot{\varphi}_{2}(0)$. Therefore $L_{g} \varphi_{i}(0) \neq 0$ for at least one $1 \leq i \leq 2$ and the item (iii) holds as well. The above analysis also shows that at any point $x \in \mathcal{M}$ that satisfies $L_{g} \varphi_{1}(x)=L_{g} \varphi_{2}(x)=0$, the pair $\left(\varphi_{1}, \varphi_{2}\right)$ fails to be an $x$-flat output. In other words, $\operatorname{Sing}\left(\varphi_{1}, \varphi_{2}\right)=\left\{x \in \mathcal{M}:\left(L_{g} \varphi_{1}(x), L_{g} \varphi_{2}(x)\right)=(0,0)\right\}$.

Now we will show how Proposition 1.3 follows from Theorem 2.2.

Proof. (of Prop. 1.3) Let $\left(\varphi_{1}, \varphi_{2}\right)$ be a pair of $x$-flat outputs at $\left(x_{0}, u_{0}\right), u_{0} \notin U_{\text {sing }}\left(x_{0}\right)$. Then $\left(\varphi_{1}, \varphi_{2}\right)$ satisfy the items (i)-(iii) of Theorem 2.2 and we can follow around $x_{0}$ the procedure described in the sufficiency part of that theorem in order to bring $\Sigma$ into the chained form $\Sigma_{\text {chain }}$ such that $z_{1}=\varphi_{1}$ and $z_{2}=\varphi_{2}+k_{0} \varphi_{1}$, where $k_{0}=-\frac{\psi_{2}}{\psi_{1}}(0) \in \mathbb{R}$ is a constant.

\subsection{Proof of Theorem 2.3}

Proof. Sufficiency: We will prove separately the cases $\operatorname{dim} M=3$ and $\operatorname{dim} M \geq 4$.

Case $(\mathbf{I}): \operatorname{dim} M=3$

Let $\varphi_{1}, \varphi_{2}$ be any functions satisfying (i) ${ }^{\prime}$ and (ii) ${ }^{\prime}$. Choose two vector fields $\tilde{g}_{1}, g_{2}$ such that $\mathcal{D}=\operatorname{span}\left\{\tilde{g}_{1}, g_{2}\right\}$, $\mathcal{L}=\operatorname{span}\left\{g_{2}\right\}$. We have $\tilde{g}_{1}\left(x_{0}\right) \notin \mathcal{L}\left(x_{0}\right)$ and thus there exits a function $\varphi_{i}$ such that $L_{\tilde{g}_{1}} \varphi_{i}\left(x_{0}\right) \neq 0$, say $L_{\tilde{g}_{1}} \varphi_{1}\left(x_{0}\right) \neq 0$. Introduce coordinates $x_{1}=\varphi_{1}, x_{2}=\varphi_{2}-\frac{L_{\tilde{g}_{1}} \varphi_{2}}{L_{\tilde{g}_{1}} \varphi_{1}}\left(x_{0}\right) \varphi_{1}$, complete them to a coordinate system $\left(x_{1}, x_{2}, x_{3}\right)$, and define $g_{1}=\frac{1}{L_{\tilde{g}_{1}} \varphi_{1}} \tilde{g}$. Then the associated control system $\dot{x}=u_{1} g_{1}(x)+u_{2} g_{2}(x)$ becomes

$$
\begin{aligned}
& \dot{x}_{1}=u_{1} \\
& \dot{x}_{2}=\psi(x) u_{1} \\
& \dot{x}_{3}=\eta(x) u_{2}
\end{aligned}
$$


where $\psi$ and $\eta$ are smooth functions satisfying $\psi\left(x_{0}\right)=0$ and $\eta\left(x_{0}\right) \neq 0$. The condition $\operatorname{dim} \mathcal{D}^{(1)}\left(x_{0}\right)=3$ implies that $g_{1}, g_{2}$ and $\left[g_{1}, g_{2}\right]$ are independent at $x_{0} \in \mathbb{R}^{3}$ and hence $\frac{\partial \psi}{\partial x_{3}}\left(x_{0}\right) \neq 0$. Replacing $x_{3}$ by $\psi$ and applying a feedback to normalize $\dot{\psi}$, we get

$$
\begin{aligned}
& \dot{x}_{1}=u_{1} \\
& \dot{x}_{2}=x_{3} u_{1} \\
& \dot{x}_{3}=u_{2}
\end{aligned}
$$

for which $\left(x_{1}, x_{2}\right)=\left(\varphi_{1}, \varphi_{2}-\frac{L_{\tilde{g}_{1}} \varphi_{2}}{L_{\tilde{g}_{1}} \varphi_{1}}\left(x_{0}\right) \varphi_{1}\right)$ is an $x$-flat output at $\left(x_{0}, u_{0}\right)$, where $u_{0}=\left(u_{10}, u_{20}\right)^{\top}$ such that $u_{10} \neq 0$, and thus $\left(\varphi_{1}, \varphi_{2}\right)$ is an $x$-flat output at $\left(x_{0}, u_{0}\right)$, where $u_{0} \neq U_{\text {sing }}^{\varphi}\left(x_{0}\right)$. Indeed, $\left(\operatorname{span}\left\{\mathrm{d} \varphi_{1}, \mathrm{~d} \varphi_{2}\right\}\right)^{\perp}=$ $\operatorname{span}\left\{\frac{\partial}{\partial x_{3}}\right\}$ and $u_{0} \neq U_{\text {sing }}^{\varphi}\left(x_{0}\right)$ is equivalent to $u_{10} \neq 0$.

Case (II): $\operatorname{dim} M \geq 4$

Take any 2-input system whose associated distribution satisfies $\operatorname{dim} \mathcal{D}^{(i)}(x)=\operatorname{dim} \mathcal{D}_{i}(x)=i+2$ everywhere in a neighborhood of $x_{0}$ and choose two functions $\varphi_{1}$ and $\varphi_{2}$ fulfilling (i) ${ }^{\prime}-(\text { iii) })^{\prime}$. We can bring $\Sigma$ to the chained form (1.3) in coordinates $\left(z_{1}, \ldots, z_{n+2}\right)$, transforming $x_{0}$ into $0 \in \mathbb{R}^{n+2}$, and its associated distribution is $\mathcal{D}=\operatorname{span}\left\{g_{1}, g_{2}\right\}$, where

$$
\begin{aligned}
& g_{1}=\frac{\partial}{\partial z_{1}}+z_{3} \frac{\partial}{\partial z_{2}}+\cdots+z_{n+2} \frac{\partial}{\partial z_{n+1}} \\
& g_{2}=\frac{\partial}{\partial z_{n+2}}
\end{aligned}
$$

The characteristic distribution $\mathcal{C}_{n-1}$ is given by $\mathcal{C}_{n-1}=\operatorname{span}\left\{\frac{\partial}{\partial z_{4}}, \ldots, \frac{\partial}{\partial z_{n+2}}\right\}$. Item (ii) $)^{\prime}$ implies that $\mathcal{C}_{n-1} \subset$ $\mathcal{L}=\left(\operatorname{span}\left\{\mathrm{d} \varphi_{1}, \mathrm{~d} \varphi_{2}\right\}\right)^{\perp}$. Indeed, if there were a vector field $f \in \mathcal{C}_{n-1}$ such that $f \notin \mathcal{L}$, then $\mathcal{D}^{(n-1)}=$ $\mathcal{L}+\operatorname{span}\{f\}$ and hence

$$
\begin{aligned}
\mathcal{D}^{(n)} & =\mathcal{D}^{(n-1)}+\left[\mathcal{D}^{(n-1)}, \mathcal{D}^{(n-1)}\right] \\
& =\mathcal{L}+\operatorname{span}\{f\}+[f, \mathcal{L}] \\
& =\mathcal{L}+\operatorname{span}\{f\} \\
& =\mathcal{D}^{(n-1)}
\end{aligned}
$$

which contradicts the condition $\operatorname{rank} \mathcal{D}^{(n)}=n+2$. Therefore $\mathcal{C}_{n-1} \subset \mathcal{L}$ holds indeed. Consequently we have $L_{c} \varphi_{i} \equiv 0$, for $i=1,2$ and any $c \in \mathcal{C}_{n-1}$, which implies that $\varphi_{i}=\varphi_{i}\left(z_{1}, z_{2}, z_{3}\right)$ for $i=1,2$. Moreover observing that $g_{2} \in \mathcal{C}_{n-1} \subset \mathcal{L}$, by item (iii) ${ }^{\prime}$ we conclude $g_{1} \notin \mathcal{L}$, which implies that there exists $\varphi_{i}, 1 \leq i \leq 2$, such that $L_{g_{1}} \varphi_{i}(0) \neq 0$, say $i=1$. In other words, we have $L_{g_{1}} \varphi_{1}(0)=\frac{\partial \varphi_{1}}{\partial z_{1}}(0) \neq 0$. By (i) ${ }^{\prime}$ we can complete $\varphi_{1}$ and $\varphi_{2}$ by a function $\varphi_{3}\left(z_{1}, z_{2}, z_{3}\right)$ such that $\left(\mathrm{d} \varphi_{1} \wedge \mathrm{d} \varphi_{2} \wedge \mathrm{d} \varphi_{3}\right)(0) \neq 0$. The remaining part of the proof follows the same line as that of Theorem 2.2 .

Necessity: Assume that $\Sigma$ is $x$-flat at $\left(x_{0}, u_{0}\right), u_{0} \notin U_{\text {sing }}\left(x_{0}\right)$, and $\left(\varphi_{1}, \varphi_{2}\right)$ is an $x$-flat output at $\left(x_{0}, u_{0}\right)$ (if $n=1$, i.e., $\operatorname{dim} M=3$, we assume that $u_{0} \notin U_{\operatorname{sing}}^{\varphi}\left(x_{0}\right)$ ), defined in a neighborhood $\mathcal{M}$ of $x_{0}$. It is well known (see $[7,13,28])$ that $\mathrm{d} \varphi_{1}\left(x_{0}\right) \wedge \mathrm{d} \varphi_{2}\left(x_{0}\right) \neq 0$. Now we will prove the item (ii) ${ }^{\prime}$. Clearly, Lemma 4.1 applies and thus there exists an open and dense subset $\tilde{\mathcal{M}} \subset \mathcal{M}$ with the properties claimed by the lemma. Around any $x \in \tilde{\mathcal{M}}$, there exists a local coordinate system $\left(z_{1}, \ldots, z_{n+2}\right)$ such that $z_{1}=\varphi_{1}, z_{2}=\varphi_{2}+k_{0} \varphi_{1}$, where $k_{0} \in \mathbb{R}$, in which $\Sigma$ takes the chained form $\Sigma_{\text {chain }}(1.3)$ and $x$ is transformed into $0 \in \mathbb{R}^{n+2}$. Then by a simple 
computation we get $\mathcal{D}^{(n-1)}=\operatorname{span}\left\{\frac{\partial}{\partial z_{n+2}}, \frac{\partial}{\partial z_{n+1}}, \ldots, \frac{\partial}{\partial z_{3}}, \frac{\partial}{\partial z_{1}}+z_{3} \frac{\partial}{\partial x_{2}}\right\}$. Hence

$$
\begin{aligned}
\mathcal{L} & =\left(\operatorname{span}\left\{\mathrm{d} \varphi_{1}, \mathrm{~d} \varphi_{2}\right\}\right)^{\perp} \\
& =\left(\operatorname{span}\left\{\mathrm{d} z_{1}, \mathrm{~d} z_{2}\right\}\right)^{\perp} \\
& =\operatorname{span}\left\{\frac{\partial}{\partial z_{n+2}}, \frac{\partial}{\partial z_{n+1}}, \ldots, \frac{\partial}{\partial z_{3}}\right\} \\
& \subset \mathcal{D}^{(n-1)} .
\end{aligned}
$$

Now consider a point $x \in \mathcal{M} \backslash \tilde{\mathcal{M}}$. The distributions $\mathcal{L}=\left(\operatorname{span}\left\{\mathrm{d} \varphi_{1}, \mathrm{~d} \varphi_{2}\right\}\right)^{\perp}$ and $\mathcal{D}^{(n-1)}$ are of constant rank and they are well defined at any point of $\mathcal{M}$. Assume that $\mathcal{L}(x)=\left(\operatorname{span}\left\{\mathrm{d} \varphi_{1}(x), \mathrm{d} \varphi_{2}(x)\right\}\right)^{\perp} \not \subset \mathcal{D}^{(n-1)}(x)$, then, because of constant ranks of $\mathcal{L}$ and $\mathcal{D}^{(n-1)}$, the inclusion does not hold at any $\tilde{x}$ in an open neighborhood $\tilde{\mathcal{O}}$ of $x$. Clearly, $\tilde{\mathcal{M}} \cap \tilde{\mathcal{O}} \neq \emptyset$ which gives a contradiction. In conclusion, we have $\mathcal{L}(x)=\operatorname{span}\left\{\mathrm{d} \varphi_{1}(x), \mathrm{d} \varphi_{2}(x)\right\}^{\perp} \subset$ $\mathcal{D}^{(n-1)}(x)$ for any point $x$ in $\mathcal{M}$. Observe that $\mathcal{D}\left(x_{0}\right) \not \subset \mathcal{L}\left(x_{0}\right)$ holds if and only if there exists a vector field $g_{1} \in \mathcal{D}$ such that $\left(L_{g_{1}} \varphi_{1}\left(x_{0}\right), L_{g_{1}} \varphi_{2}\left(x_{0}\right)\right) \neq(0,0)$, which is just the item (iii) of Theorem 2.2. This shows the equivalence of the two singular loci defined in Theorem 2.2 and Theorem 2.3 and proves, due to Theorem 2.2, the necessity of (iii)'.

\subsection{Proof of Theorem 2.7}

Proof. The results of Sections 2.2 and 2.3 show that for a given arbitrary smooth function $\varphi_{1}$ such that $L_{c} \varphi_{1}=0$, for any $c \in \mathcal{C}_{n-1}$, and $L_{g} \varphi_{1}\left(x_{0}\right) \neq 0$, there always exists a function $\varphi_{2}$, independent with $\varphi_{1}$, such that $\left(\varphi_{1}, \varphi_{2}\right)$ is an $x$-flat output of $\Sigma$ at $\left(x_{0}, u_{0}\right), u_{0} \notin U_{\text {sing }}\left(x_{0}\right)$. By Proposition 1.3, we can introduce new coordinates by $z_{1}=\varphi_{1}, z_{2}=\varphi_{2}+k_{0} \varphi_{1}$, where $k_{0} \in \mathbb{R}$, and complete them to a coordinate system $\left(z_{1}, \ldots, z_{n+2}\right)$ in which our original system $\Sigma$ takes, via a feedback transformation, the chained form $\Sigma_{\text {chain }}$, given by (1.3). Suppose that there exists another function $\tilde{\varphi}_{2}$ such that $\left(\varphi_{1}, \tilde{\varphi}_{2}\right)=\left(z_{1}, \tilde{\varphi}_{2}\right)$ is an $x$-flat output of $\Sigma$ at $\left(x_{0}, u_{0}\right), u_{0} \notin U_{\text {sing }}\left(x_{0}\right)$. Clearly, $\left(\varphi_{1}, \tilde{\varphi}_{2}\right)$ is also an $x$-flat output of $\Sigma_{\text {chain }}$ at $\left(0, v_{0}\right), v_{0} \notin U_{\text {sing }}(0)$. Take $g=\frac{\partial}{\partial z_{1}}+z_{3} \frac{\partial}{\partial z_{2}}+\cdots+z_{n+2} \frac{\partial}{\partial z_{n+1}}$ and let $\mathcal{C}_{n-1}$ be the characteristic distribution of $\mathcal{D}^{(n-1)}$, where $\mathcal{D}$ is the associated distribution of $\Sigma_{\text {chain. }}$ By the result of Sections 2.2 and 2.3 , the function $\tilde{\varphi}_{2}$ must satisfy the equations

$$
\begin{aligned}
& L_{c} \tilde{\varphi}_{2}=0, \quad \forall c \in \mathcal{C}_{n-1}, \\
& L_{v} \tilde{\varphi}_{2}=0 .
\end{aligned}
$$

where $v=\left(L_{g} \varphi_{1}\right)\left[c_{n-1}, g\right]-\left(L_{\left[c_{n-1}, g\right]} \varphi_{1}\right) g$ and $c_{n-1}=\frac{\partial}{\partial z_{4}}$. Solving the above system of equations, we get $\tilde{\varphi}_{2}=\left\{\tilde{\varphi}_{2}\left(z_{1}, z_{2}\right): \frac{\partial \tilde{\varphi}_{2}}{\partial z_{2}}(0) \neq 0\right\}$. Thus

$$
\begin{aligned}
\operatorname{span}\left\{\mathrm{d} \varphi_{1}, \mathrm{~d} \varphi_{2}\right\}(z) & =\operatorname{span}\left\{\mathrm{d} z_{1}, \mathrm{~d} z_{2}\right\}(z) \\
& =\operatorname{span}\left\{\mathrm{d} z_{1}, \frac{\partial \tilde{\varphi}_{2}}{\partial z_{1}}(z) \mathrm{d} z_{1}+\frac{\partial \tilde{\varphi}_{2}}{\partial z_{2}}(z) \mathrm{d} z_{2}\right\}(z) \\
& =\operatorname{span}\left\{\mathrm{d} \varphi_{1}, \mathrm{~d} \tilde{\varphi}_{2}\right\}(z),
\end{aligned}
$$

for any $z$ in a neighborhood of 0 . Correspondingly, for the original system $\Sigma$, the above equality is true for any point in a neighborhood of $x_{0}$.

\section{REFERENCES}

[1] E. Aranda-Bricaire, C.H. Moog and J.-B. Pomet, Infinitesimal Brunovský form for nonlinear systems with applications to Dynamic Linearization, Geometry in nonlinear control and differential inclusions 32, edited by B. Jakuczyk, W. Respondek and T. Rzeżuchowski. Banach Center Publications, Warsaw (1995) 19-33.

[2] R. Bryant, S.-S. Chern, R. Gardner, H. Goldschmidt and P. Griffiths, Exterior Differential Systems. Mathematical Sciences Research Institute Publications, Springer-Verlag, New York (1991). 
[3] E. Cartan, Sur l'équivalence absolue de certains systèmes d'équations différentielles et sur certaines familles de courbes, Bulletin de la Société Mathématique de France 42, Cuvres complètes 2. Part. II, Gauthiers-Villars, Paris (1914) 12-48.

[4] M. Cheaito and P. Mormul, Rank-2 distributions satisfying the Goursat condition:all their local models in dimension 7 and 8. ESAIM: COCV 4 (1999) 137-158.

[5] M. Fliess, J. Lévine, P. Martin and P. Rouchon, Sur les systèmes non linéaires différentiellement plats. C. R. Acad. Sci. 315 (1992) 619-624.

[6] M. Fliess, J. Lévine, P. Martin and P. Rouchon, Flatness and defect of nonlinear systems: Introductory theory and examples. Int. J. Control 61 (1995) 1327-1361.

[7] M. Fliess, J. Lévine, P. Martin and P. Rouchon, A Lie-Bäcklund approach to equivalence and flatness of nonlinear systems. IEEE Trans. Automat. Control 61 (1999) 1327-1361.

[8] A. Giaro, A. Kumpera and C. Ruiz, Sur la lecture correcte d'un resultat d'Élie Cartan. C. R. Acad. Sci. Paris 287 (1978) 241-244.

[9] E. Goursat, Leçons sur le problème de Pfaff. Hermann, Paris (1923).

[10] D. Hilbert, Über den Begriff der Klasse von Differentialgleichungen. Math. Ann. 73 (1912) 95-108.

[11] A. Isidori. Nonlinear Control Systems, 3rd edition. Springer-Verlag, London (1995).

[12] A. Isidori, C.H. Moog and A. de Luca. A sufficient condition for full linearization via dynamic state feedback, in Proc. 25th IEEE Conf. on Decision \& Control. Athens (1986) 203-207.

[13] B. Jakubczyk, Invariants of dynamic feedback and free systems, in Proceedings of the European Control Conference. Groningen (1993) 1510-1513.

[14] F. Jean, The car with $n$ trailers: Characterisation of the singular configurations. ESAIM: COCV 1 (1996) 241-266.

[15] A. Kumpera and C. Ruiz, Sur l'équivalence locale des systèmes de Pfaff en drapeau, in Monge-Ampère equations and related topics, edited by F. Gherardelli. Instituto Nazionale di Alta Matematica Francesco Severi, Rome (1982) 201-247.

[16] J.P. Laumond, Controllability of a multibody robot. IEEE Trans. Robot. Autom. 9 (1991) 755-763.

[17] J.P. Laumond, Robot Motion Planning and Control, Lecture Notes on Control and Information Sciences 229. Springer-Verkag, New York (1997).

[18] Z. Li and J.F. Canny Eds., Nonholonomic Motion Plannging. Internqtional Series in Engineering and Computer Sciences, Kluwer, Dordrecht (1992).

[19] P. Martin and P. Rouchon, Feedback linearization and driftless systems. CAS internal report No. 446, École des Mines (1993).

[20] P. Martin and P. Rouchon, Feedback linearization and driftless systems. Math. Contr. Signals Syst. 7 (1994) $235-254$.

[21] P. Martin, R.M. Murray and P. Rouchon, Flat systems, in Mathematical Control Theory, Part 2, ICTP Lecture Notes 8, edited by A.A. Agrachev. ICTP Publications, Trieste (2002) 705-768.

[22] P. Mormul, Goursat flags: classification of codimension-one singularities. J. Dyn. Control Syst. 6 (2000) 311-330.

[23] R. Murray, Nilpotent bases for a class of nonintegrable distributions with applications to trajectory generation for nonholonomic systems. Math. Control Signals Syst. 7 (1994) 58-75.

[24] R. Murray and S. Sastry, Nonholonomic motion planning: Steering using sinusoids. IEEE Trans. Autom. Control 38 (1993) $700-716$.

[25] W. Pasillas-Lépine and W. Respondek, On the geometry of control systems equivalent to canonical contact systems: regular points, singular points and flatness, Proceedings of the 39th IEEE Conference of Decision and Control. Sydney, Australia (2000) 5151-5156.

[26] W. Pasillas-Lépine and W. Respondek, On the geometry of Goursat structures. ESAIM: COCV 6 (2001) 119-181.

[27] P.S. Pereira da Silva and C. Corrêa Filho, Relative flatness and flatness of implicit systems. SIAM J. Control Optim. 39 (2001) 1929-1951.

[28] J.-B. Pomet, A differential geometric setting for dynamic equivalence and dynamic linearization, in Geometry in Nonlinear Control and Differential Inclusions 32, edited by B. Jakubczyk, W. Respondek and T. Rzeżuchowski. Banach Center Publications, Warsaw (1995) 319-339.

[29] W. Respondek, Symmetries and minimal flat outputs of nonlinear control systems, in New Trends in Nonlinear Dynamics and Control, and their Applications, Lecture Notes on Control and Information Sciences 295, edited by W. Kang, M. Xiao and C. Borges. Springer Verlag, Berlin, Heidelberg (2003) 65-86.

[30] O.J. Sørdalen, Conversion of the kinematics of a car with $\mathrm{n}$ trailers into a chained form, Proceeding of 1993 International Conference on Robotics and Automation, Atlanta, CA (1993) 382-387.

[31] M. van Nieuwstadt, M. Rathinam and R.M. Murray, Differential Flatness and Absolute Equivalence of Nonlinear Control Systems. SIAM J. Control Optim. 36 (1998) 1225-1239.

[32] E. von Weber, Zur Invariantentheorie der Systeme Pfaff'scher Gleichungen. Berichte Verhandlungen der Koniglich Sachsischen Gesellshaft der Wissenshaften Mathematisch-Physikalische Klasse, Leipzig 50 (1898) 207-229. 